\title{
A Complementarity Model for Electric Power Transmission-Distribution Coordination Under Uncertainty
}

Hermann, Alexander Niels August; Jensen, Tue Vissing; Østergaard, Jacob; Kazempour, Jalal

Published in:

European Journal of Operational Research

Link to article, DOI:

10.1016/j.ejor.2021.08.018

Publication date:

2022

Document Version

Peer reviewed version

Link back to DTU Orbit

Citation (APA):

Hermann, A. N. A., Jensen, T. V., Østergaard, J., \& Kazempour, J. (2022). A Complementarity Model for Electric Power Transmission-Distribution Coordination Under Uncertainty. European Journal of Operational Research, 299(1), 313-329. https://doi.org/10.1016/j.ejor.2021.08.018

\section{General rights}

Copyright and moral rights for the publications made accessible in the public portal are retained by the authors and/or other copyright owners and it is a condition of accessing publications that users recognise and abide by the legal requirements associated with these rights.

- Users may download and print one copy of any publication from the public portal for the purpose of private study or research.

- You may not further distribute the material or use it for any profit-making activity or commercial gain

- You may freely distribute the URL identifying the publication in the public portal 


\title{
A Complementarity Model for Electric Power Transmission-Distribution Coordination Under Uncertainty
}

\author{
Alexander Hermann, Tue Vissing Jensen, Jacob Østergaard, and Jalal Kazempour \\ Department of Electrical Engineering, Technical University of Denmark, Kongens Lyngby, Denmark
}

\begin{abstract}
The growing penetration of stochastic renewable energy sources increases the need for operational flexibility to cope with imbalances. Existing proposals for flexibility procurement are envisioning markets where the transmission system operator (TSO) can access flexible resources located at the distribution system operator (DSO)-level and vice versa, but the coordination between these two entities is a matter of active research. We consider two trading floors, i.e., day-ahead and real-time markets, and propose a method for day-ahead coordination on how to share flexible resources, described as a complementarity model. The proposed coordination approach is to optimize prices and capacity limits at the physical interface of TSO and DSO, the so-called "coordination variables". For given values of these variables, the DSO pre-qualifies the participation of DSO-level resources in the day-ahead market by capping their quantity bids. This way, the DSO ensures that the constraints of its system, modeled by a conic program, will be respected. Pursuing computational tractability, we decompose the model using a multi-cut Benders' decomposition approach. It separates the conic modeling of real-time power flows under each scenario from the mixed-integer linear formulation of the day-ahead market-clearing problem. We quantify the potential benefit of the proposed coordination method in terms of improved social welfare. Using an ex-post out-ofsample simulation, the performance of the proposed coordination method is assessed against two benchmarks: $(i)$ a fully uncoordinated scheme which obtains a lower bound for the expected social welfare, and (ii) an ideal benchmark which co-optimizes the TSO and DSO problems, providing an upper bound for the expected social welfare.
\end{abstract}

Keywords: OR in energy; TSO-DSO coordination; Stochastic conic programming; Bi-level optimization; Multi-cut Benders' decomposition

\section{Introduction}

\subsection{On the need for flexibility}

The European day-ahead electricity market uses a simplified representation of transmission constraints. This market solely considers network constraints that restrict power exchange among pre-defined market areas, the so-called bidding zones (Ehrenmann \& Smeers, 2005). Each zone is defined as a geographical area representing a part of or an entire country. Since zonal markets discard the intra-zone network constraints, the market participants within a zone are able to exchange 
energy without being enforced by any transmission constraint ${ }^{1}$ (Aravena \& Papavasiliou, 2017). The clearing outcomes of zonal electricity markets are not necessarily feasible in the operational stage. In every zone, it is the responsibility of the corresponding Transmission System Operator (TSO) to restore the feasibility and operate the power system safely on high-voltage transmission level, while respecting exchange limits between the zones. To make the market outcomes feasible, the TSO requires flexibility ${ }^{2}$. In addition, the TSO is in charge of balancing any mismatch between demand and supply in the real-time operation, a task for which flexibility is also required. Maintaining this balance becomes more challenging for TSOs as the share of renewable energy sources with uncertain and variable supply grows. The bottom line is that TSOs need to access flexibility, and this need in terms of the volume and the diversity of flexible resources is increasing (Villar et al., 2018).

Among all potential sources of flexibility, it is foreseen that distributed energy resources will be able to meet a significant part of the flexibility needs of TSOs (Callaway \& Hiskens, 2011; Østergaard et al., 2021). Two examples of such resources are controllable electric loads and smallscale energy storage systems. These resources are often located at medium-voltage and radial low-voltage feeders of distribution systems, which are outside the operational domain of the TSO. Each distribution system is operated by the corresponding Distribution System Operator (DSO). Note that the TSO and the DSO are separate and independent entities with their own distinct objectives, although their underlying systems are physically connected.

\subsection{TSO-DSO coordination: why and how?}

The critical issue is that the activation of distributed energy resources by the TSO to produce flexibility will impact the distribution grid operation. It is often the case that the flexibility activation violates the operational constraints of distribution systems, such as nodal voltage or line capacity limits (Verzijlbergh et al., 2014). This is exactly the case where a conflict between TSO and DSO may happen, since the DSO is in charge of the safe operation of its corresponding distribution system. To resolve such a potential confliction, coordination between TSO and DSO, the so-called "TSO-DSO coordination", is essential ${ }^{3}$ (Gerard et al., 2018; Hadush \& Meeus, 2018).

By TSO-DSO coordination, and in general the coordination between any two or multiple entities, we refer to a mechanism that improves the overall system efficiency by allowing each entity to use the resources belonging to the system of the other entities, while respecting all operational restrictions and current regulations. The coordination between entities could be achieved through operational mechanisms (Silva et al., 2018), financial instruments (Schwele et al., 2021), and/or local information channels (Dahleh et al., 2016). All these coordination mechanisms aim to enhance the information flow, either directly or indirectly, among entities. In a market context, the information among entities could be indirectly exchanged through bids, market products, or players at the interface of entities, while respecting the underlying regulations. The information

\footnotetext{
${ }^{1}$ The central western European power system uses a revisited zonal market design, the so-called flow-based market coupling model (Van den Bergh et al., 2016), aiming to improve the efficiency of the zonal design by representing the intra-zone network constraints to some extent by including additional polyhedral constraints on the zonal net injections (Lété \& Papavasiliou, 2021).

${ }^{2}$ By flexibility, we refer to the capability of an asset, e.g., a flexible generator or a controllable load, to modify its power dispatch, either production or consumption level, if requested by a system operator (Zhao et al., 2016).

${ }^{3} \mathrm{~A}$ transmission system may connect to several distribution systems, and therefore a TSO may need to have a coordination mechanism with several DSOs at the same time.
} 
flow will eventually allow entities to exploit the available resources in a way that benefits not only individuals but also the whole system.

\subsection{State of the art and the research gap}

There are various proposals for the TSO-DSO coordination in the existing literature. In the following, we group these proposals in four distinct categories:

The first category of proposals suggests a disruptive solution, built upon the co-optimization of TSO and DSO operational problems. The regulatory challenge is that these proposals require a single entity (representing both TSO and DSO) to solve the co-optimization problem, which is against the current practice under which TSO and DSO operate independently. This co-optimization problem can be viewed as an approach that provides the ideal coordination, so it can serve as an ideal benchmark. Aligned with this category, Caramanis et al. (2016) and Mohammadi et al. (2019) cooptimize TSO and DSO operation using distributed optimization techniques. This solution leads to an iterative optimization mechanism, and still does not comply with current regulations (Gerard et al., 2016).

The second category of proposals offers a direct exchange of information between TSO and DSO. One possible method for the DSO is to forecast the grid loading and share the feasible injection region at the interface with the TSO (Gonzalez et al., 2018; Silva et al., 2018; Yuan \& Hesamzadeh, 2017). This method requires the TSO to incorporate the feasible region of every DSO, which it may be unwilling to do due to the large scale of the operational problem. Note that sharing the feasible space requires bi-directional exchanges of messages, because first the DSO must receive information about prices at the interface, and subsequently communicate the feasible space to the TSO.

The third category of proposals is based on designing a local flexibility market, where the DSO can procure flexibility to meet the local network constraints. The remaining flexibility in the distribution system can then be exploited by the TSO. Various local market designs are proposed by Zhang et al. (2014); Ströhle \& Flath (2016); Bai et al. (2018); Morstyn et al. (2019); Vicente Pastor et al. (2019), Laur et al. (2020) and Heinrich et al. (2021).

The fourth category of proposals allows the DSO to optimally limit the quantity bids of local distributed energy resources or their aggregations to wholesale or TSO-level markets in order to meet the local grid constraints. This is a framework where the DSO restricts the participation of local distributed energy resources in the day-ahead pool and the TSO-level flexibility market. Aligned with this category, the European SmartNet project (Gerard et al., 2016; The SMARTNET Project Final Results, 2019) and the Danish DREM project (Brath et al., 2019) have proposed several methods where the DSO constrains the bids of distributed energy resources to wholesale markets. This can be viewed as the pre-qualification of bids by the DSO, allowing the qualified distributed energy resources to participate in the wholesale and TSO-level flexibility markets.

The common point of all proposals in the above four categories is that the TSO and DSO coordinate their flexibility procurement at a point of time after the day-ahead market, but not before it. However, in most countries the bulk of electricity is traded in the day-ahead market, and therefore, any coordination that is included in the day-ahead market is expected to increase the social welfare ${ }^{4}$ of the whole system. In addition, the majority of the existing proposals are

\footnotetext{
${ }^{4}$ Social welfare, also known as market surplus, is the total utility of demands minus the total operational cost of the system, including the production cost of conventional and renewable power units as well as the cost for involuntarily load shedding, the so-called load curtailment cost.
} 
not congruent with current European electricity market regulations. Recall that the European day-ahead electricity market uses a zonal market design with a simplified network representation, and the proposed TSO-DSO coordination mechanisms for European power systems are expected to be aligned with the zonal market design.

\subsection{Our research questions and contributions}

Given the context above, a relevant research question is how a day-ahead coordination between the TSO and DSOs could take place. To enable such a coordination, our proposal is to focus on the interface ${ }^{5}$ between the TSO and each DSO, and treat the flow capacity limit and the electricity price at the interface as "coordination variables" in the day-ahead stage. By optimizing these coordination variables, the access of the TSO to DSO-level flexible resources ${ }^{6}$ can be adjusted.

It is worth mentioning that the day-ahead market is a purely financial trading floor, in the sense that the actual power delivery does not occur in that time stage. Therefore, the system operators do not necessarily require to impose in the day-ahead stage the real physical capacity limits of the interface, as long as such limits will be imposed later in the subsequent markets, e.g., in intra-day or real-time markets. However, we need a holistic model such that it optimizes not only the day-ahead coordination variables, but also accounts for any additional operational cost in subsequent markets incurred by enforcing the real physical limits. In such a framework, the interface capacity limits in the day-ahead stage can be higher than, equal to, or lower than the real physical limits.

The proposed coordination mechanism raises an institutional question: who is in charge of optimizing the coordination variables? We introduce a new agent to fulfill this task, called the "interface optimizer", who is a non-profit oriented agent for TSO-DSO coordination ${ }^{7}$. There is no such an entity in current practice, and we do not rush regulators and industry to establish it. Instead, we see our proposed method as a tool to show the maximum potential room for TSODSO coordination by optimizing the interface characteristics at the day-ahead stage, which is a completely new concept. Our proposed tool shows that it might be possible to improve the sectoral coordination between TSO and DSOs, without a need for extreme disruptive solutions. It is outside the scope of this work how the TSO and DSOs can practically implement this idea while respecting their current sectoral policies and market regulations.

The next question raised is how to develop a proper mathematical tool for the interface optimizer to determine the optimal coordination variables, which can be used as a reference for a future practical coordination mechanism. The interface optimizer seeks to maximize the expected social welfare of the entire system, including both transmission and distribution levels, and in both day-ahead and real-time trading stages. We propose a bi-level optimization model, whose leader is the interface optimizer. The followers are DSOs as well as day-ahead and real-time markets. The potential uncertainties, e.g., wind power generation in both DSO and TSO levels, are modeled by a finite number of scenarios. Under some assumptions, we mathematically prove that the first stage in the resulting stochastic bi-level model can be reduced with no approximation. To ease

\footnotetext{
${ }^{5}$ Interface, also known as the point of common coupling (PCC), refers to the node connecting a distribution system to the transmission grid.

${ }^{6}$ We use distributed energy resources and DSO-level flexible resources interchangeably.

${ }^{7}$ The extension of this idea by considering the interface optimizer as a merchant for-profit agent might be possible, but it will likely reduce the efficiency of the proposed coordination mechanism. We leave such a study for future works.
} 
the computational burden, we decompose the reduced bi-level problem using a multi-cut Benders' decomposition algorithm. A side benefit of this decomposition is that it avoids solving a mixedinteger second-order cone program, and separates it to a mixed-integer linear problem and a set of convex and continuous Second-Order Cone Programming (SOCP) problems. We quantify the potential benefit of the proposed coordination method in terms of improved social welfare. Using an ex-post out-of-sample simulation, the performance of the proposed coordination method is assessed against two benchmarks: $(i)$ a fully uncoordinated mechanism which obtains a lower bound for the expected social welfare, and $(i i)$ an ideal benchmark which co-optimizes the TSO and DSO problems, obtaining an upper bound for the expected social welfare.

\subsection{Paper organization}

The paper is structured as follows: Section 2 details the proposed coordination method. Section 3 provides the proposed stochastic bi-level model. Section 4 describes the implementation of Benders' decomposition approach, and defines two benchmark models. In Section 5, two case studies are presented. The first one is a small illustrative case study with a three-node transmission network and three simple DSO networks with two nodes each. Subsequently, a larger case study is presented which is based on a modified IEEE 24-node network, including one TSO and five DSOs. Section 6 summarizes the findings, discusses their influence on potential practical coordination mechanisms, and provides a few potential directions for future works. Finally, two appendices provide some details about the scenario generation method and the large-scale case study.

\section{The proposed coordination mechanism}

\subsection{Coordination via bi-level programming: state of the art}

We model the decision-making problem of the interface optimizer to determine the coordination variables as a Stackelberg game, resulting in a bi-level optimization problem (Dempe, 2002). The generic theoretical foundations of bi-level programming and a review on its applications to electric power systems can be found in Gabriel et al. (2012) and Pozo et al. (2017).

As one of the inspiring works in the literature in terms of the applications of bi-level programming for coordination purposes, Morales et al. (2014) propose a stochastic bi-level model to improve the expected social welfare by improving the temporal coordination between day-ahead and real-time markets. The proposed model in Morales et al. (2014) considers a deterministic day-ahead market including network constraints, takes the available probabilistic forecast of renewables in the day-ahead stage as input data, and determines the optimal cap on the day-ahead schedule of renewables. The determination of such a cap is not trivial, since the optimal cap is not necessarily identical to the installed capacity of the renewable source or the expected value (or maximum or minimum) of its production forecast. We go beyond Morales et al. (2014) in two ways. First, the day-ahead market in our model is not only deterministic but also excludes the network constraints which is aligned with the current practice in Europe. Second, our model offers a solution for a broader coordination in both temporal and sectoral angles (temporal coordination between day-ahead and real-time markets and sectoral coordination between the TSO and DSOs). Similar to Morales et al. (2014), the coordination tool in our work is through imposing optimal caps, although the nature of such caps in these two research works is different. While Morales et al. (2014) impose caps on the day-ahead schedule of renewables, we determine optimal caps on the participation of DSO-level flexible resources in the TSO-level markets. Our numerical results in 
Section 5 will illustrate the success of those caps to implicitly make the deterministic copper-plate day-ahead market aware of uncertainties and network constraints.

As other relevant papers in the literature, Jensen et al. (2018) determine the optimal dayahead limits for energy trading among zones through a stochastic bi-level model. Dvorkin et al. (2019a) explore the optimal allocation of reserves in electricity markets through a bi-level approach. Delikaraoglou \& Pinson (2019) develop a bi-level model to optimally coordinate the reserve capacity allocation of High-Voltage Direct Current (HVDC) lines among zones. Pursuing improved sectoral coordination among different energy systems, Ordoudis et al. (2020) propose two stochastic bi-level models for coordination between power and natural gas systems through optimizing the gas price and the gas volume availability in the electricity market. In a similar direction, Mitridati et al. (2020) propose a set of stochastic bi-level models for coordination of power and district heating systems.

\subsection{Mathematical description of a bi-level problem with single leader and multiple sequential fol- lowers}

Before introducing our proposed bi-level model for TSO-DSO coordination, let us provide a general mathematical description for a specific bi-level model that contains a single leader and three sequential followers. Our proposed bi-level model has the same mathematical structure.

Let $\mathbf{x}, \mathbf{y}_{1}, \mathbf{y}_{2}$, and $\mathbf{y}_{3}$ denote the decision variable sets of the leader, follower 1 , follower 2 , and follower 3, respectively. The leader makes decision $\mathbf{x}$ and three followers respond to it. By sequential followers, we imply that there is a decision sequence among three followers. This is detailed in the following.

The leader solves the optimization problem $\mathcal{P}^{\mathrm{L}}\left(\mathbf{x}, \mathbf{y}_{1}, \mathbf{y}_{2}, \mathbf{y}_{3}\right)$ to make optimal decision $\mathbf{x}$. The responses of followers, i.e., $\mathbf{y}_{1}, \mathbf{y}_{2}$, and $\mathbf{y}_{3}$ impact the leader's problem. For given decision $\mathbf{x}$ of the leader, follower 1 determines the optimal response $\mathbf{y}_{1}$ by optimizing problem $\mathcal{P}^{\mathrm{F} 1}\left(\mathbf{x}, \mathbf{y}_{1}\right)$. Then, for given decision $\mathbf{x}$ of the leader and response $\mathbf{y}_{1}$ of follower 1, follower 2 solves the optimization problem $\mathcal{P}^{\mathrm{F} 2}\left(\mathbf{x}, \mathbf{y}_{1}, \mathbf{y}_{2}\right)$ to find the optimal response $\mathbf{y}_{2}$. Finally, follower 3 treats $\mathbf{x}, \mathbf{y}_{1}$, and $\mathbf{y}_{2}$ as given values, and optimizes problem $\mathcal{P}^{\mathrm{F} 3}\left(\mathbf{x}, \mathbf{y}_{1}, \mathbf{y}_{2}, \mathbf{y}_{3}\right)$ to determine the optimal response $\mathbf{y}_{3}$. The resulting bi-level model is $\mathcal{P}^{\mathrm{L}}\left(\mathbf{x}, \mathbf{y}_{1}, \mathbf{y}_{2}, \mathbf{y}_{3}\right)$ constrained by three optimization problems $\mathcal{P}^{\mathrm{F} 1}\left(\mathbf{x}, \mathbf{y}_{1}\right), \mathcal{P}^{\mathrm{F} 2}\left(\mathbf{x}, \mathbf{y}_{1}, \mathbf{y}_{2}\right)$, and $\mathcal{P}^{\mathrm{F} 3}\left(\mathbf{x}, \mathbf{y}_{1}, \mathbf{y}_{2}, \mathbf{y}_{3}\right)$. Let us assume all three optimization problems of the followers are convex.

To solve this bi-level problem at once, one needs to replace the optimization problems of three followers by their corresponding optimality Karush-Kuhn-Tucker (KKT) conditions. When deriving the KKT conditions associated with problem $\mathcal{P}^{\mathrm{F} 1}\left(\mathbf{x}, \mathbf{y}_{1}\right)$, decision $\mathbf{x}$ is treated as a parameter, such that the only primal variable is $\mathbf{y}_{1}$. Similarly, the KKT conditions of problem $\mathcal{P}^{\mathrm{F} 2}\left(\mathbf{x}, \mathbf{y}_{1}, \mathbf{y}_{2}\right)$ can be derived, treating $\mathbf{x}$ and $\mathbf{y}_{1}$ as exogenous parameters while $\mathbf{y}_{2}$ is the sole primal variable. Finally, we derive the KKT conditions of problem $\mathcal{P}^{\mathrm{F} 3}\left(\mathbf{x}, \mathbf{y}_{1}, \mathbf{y}_{2}, \mathbf{y}_{3}\right)$, where the sole primal variable is $\mathbf{y}_{3}$.

The above mathematical process yields a complementarity problem, comprising of the leader's optimization problem $\mathcal{P}^{\mathrm{L}}\left(\mathbf{x}, \mathbf{y}_{1}, \mathbf{y}_{2}, \mathbf{y}_{3}\right)$ constrained by the collection of KKT conditions associated with three problems $\mathcal{P}^{\mathrm{F} 1}\left(\mathbf{x}, \mathbf{y}_{1}\right), \mathcal{P}^{\mathrm{F} 2}\left(\mathbf{x}, \mathbf{y}_{1}, \mathbf{y}_{2}\right)$, and $\mathcal{P}^{\mathrm{F} 3}\left(\mathbf{x}, \mathbf{y}_{1}, \mathbf{y}_{2}, \mathbf{y}_{3}\right)$. The variables of this complementarity problem are $\mathbf{x}, \mathbf{y}_{1}, \mathbf{y}_{2}, \mathbf{y}_{3}$ as well as all dual variables within the KKT conditions of followers. A solution to this complementarity problem is indeed a solution to the original bi-level problem. 


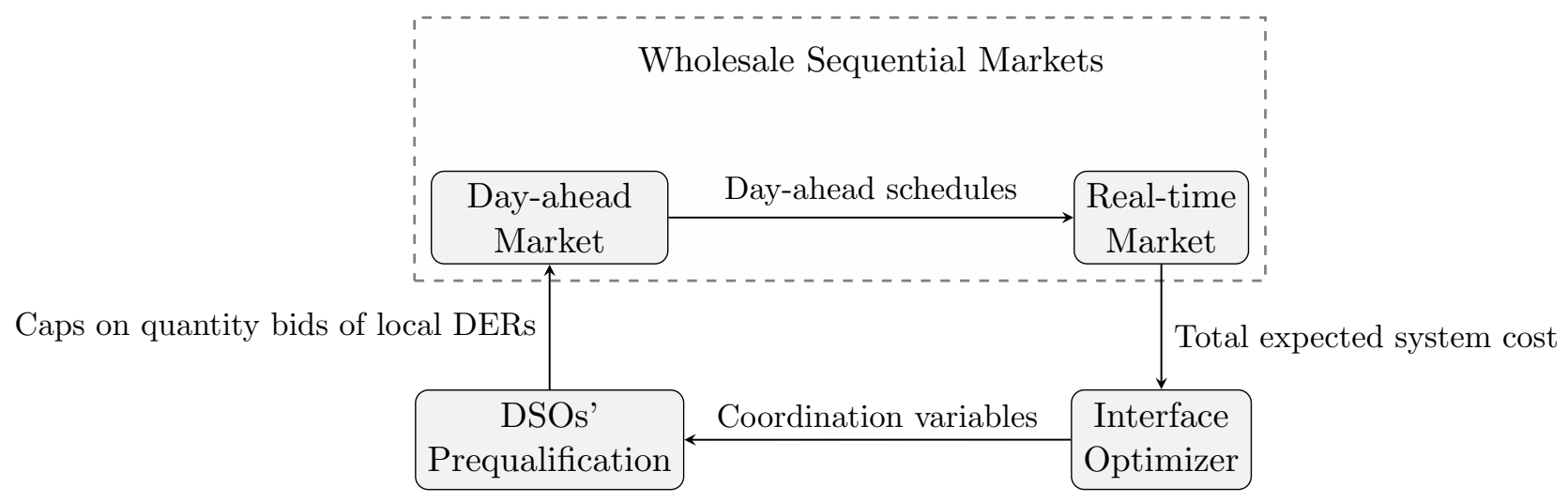

Figure 1: The conceptual passing of information between different markets and agents involved in the coordination via the proposed interface optimizer. DER: distributed energy resource.

\subsection{TSO-DSO coordination as a bi-level problem}

In our proposed bi-level optimization problem, the interface optimizer is the single leader, while three sequential followers are ( $i)$ DSOs, $(i i)$ the day-ahead market, and (iii) real-time markets. This proposed setup respects the paradigm of a two-stage market clearing, i.e., the day-ahead market and a series of real-time re-dispatches ${ }^{8}$. Although the interface optimizer solves this problem as a whole, it is essential to model the sequences in the decision-making process of three followers. Otherwise, the interface optimizer will fail in properly modeling the market interactions. The interactions of agents and markets in the proposed bi-level model are schematically illustrated in Fig. 1, and explained below by four steps:

Step 1) Leader's problem: Before the day-ahead market clearing, the interface optimizer as the leader determines the optimal values of coordination variables at each interface, i.e., prices and flow capacity limits. The interface optimizer aims to maximize the total expected social welfare of the whole system, containing TSO and DSO levels, and including both day-ahead and real-time trading stages.

Step 2) First follower's problem: Given the leader's decisions in Step 1, i.e., prices and power flow limits at the interfaces and still before the day-ahead market clearing, each DSO as the first follower puts a cap on the production or the consumption quantity that each distributed energy resource, or their aggregation, located at its domain can bid to the day-ahead market. We refer to this stage as the DSOs' pre-qualification.

Step 3) Second follower's problem: Given the quantity bids of distributed energy resources in Step 2, the day-ahead market-clearing operator as the second follower clears the market.

Step 4) Third follower's problem: Following the day-ahead market in Step 3, the real-time market operator as the third follower clears the market to adjust potential imbalances caused by the stochastic renewable power generation.

\footnotetext{
${ }^{8}$ This includes any mechanism that changes the day-ahead dispatches.
} 
The day-ahead market in Step 3, aligned with the current practice in the real-world electricity markets, is cleared in a deterministic fashion. This implies that the production quantity bid of each weather-dependent renewable power unit, e.g., a wind farm, is given by a single-point value, i.e., the mean of its production forecast. The day-ahead market in Step 3 is considered to follow an energy-only mechanism, in the sense that other market products, e.g., operating reserves, are excluded.

The real-time market in Step 4 is envisioned as a subsequent market that changes the day-ahead dispatch, depending on the availability of the updated forecast. This re-dispatch generally incurs an additional operational cost. Note that up- and down-regulation services ${ }^{9}$ to be provided by flexible resources in the real-time market may have different costs.

Recall that the whole bi-level model is solved by the interface optimizer at a point in time before the day-ahead market clearing, and the realization of renewable power generation is uncertain at that stage. This uncertainty is modeled by a finite number of scenarios ${ }^{10}$. Each scenario contains a different magnitude of imbalance between the day-ahead quantity bid of renewable power units and their real-time production in the underlying scenario. Therefore, in Step 4, we consider one realtime market-clearing problem for each renewable power scenario. These scenarios are generated based on the available forecast information at a point in time before the day-ahead market clearing, when the interface optimizer solves the proposed bi-level problem. The mean of renewable power generation over scenarios to be included in Step 4 is identical to the day-ahead quantity bid of renewable power units in Step 3. Although the proposed model depends on the used scenarios and a thorough definition of who generates them is usually pertinent, we here consider them as an external parameter. However, we will assess later in Section 5 the quality of coordination decisions made based on the given set of scenarios using an ex-post out-of-sample analysis. In our proposed model, wind farms as renewable power units bid their production at zero price, and the wind power can be freely spilled if required ${ }^{11}$.

We take the same view on network modeling as Caramanis et al. (2016) and Le Cadre et al. (2019). The power flow across the high-voltage transmission network within the operational domain of the TSO is modeled by a linear lossless power flow approximation. Although this approximation generally works properly for transmission networks, it is not necessarily a suitable model to represent power flow equations in the low-voltage distribution systems within the operational domain of the DSOs. The power losses, reactive power flow, and voltage constraints are crucial aspects in the operation of distribution systems, and they cannot be discarded. This paper determines the power flow in the radial low-voltage distribution feeders using a convex relaxation of the Alternating Current (AC) power flow equations, accounting for losses, voltage limits, and both active and reactive power flows. Specifically, we use a conic relaxation of AC power flow in the radial distribution systems (Farivar \& Low, 2013), resulting in a second-order cone program ${ }^{12}$.

\footnotetext{
${ }^{9}$ In operating conditions with a renewable power deficit, a flexible generator increases its production or a controllable demand decreases its consumption to provide up-regulation services. Similarly, for conditions with a renewable power excess, the flexible generator reduces its production or the controllable demand increases its consumption to provide down-regulation services.

${ }^{10}$ Without loss of generality, we consider the renewable power generation as the only source of uncertainty. Other potential sources of uncertainty can be modeled in the same manner, but at the expense of increased scenarios. We use a scenario generation method that correlates geographically close renewable power units. More information on our scenario generation method can be found in Appendix A.

${ }^{11}$ This operational action is referred to as wind spillage. It occurs when there is a potential for additional wind power generation, but it cannot be realized due to the lack of flexible resources to provide down-regulation services.

${ }^{12}$ Further details on different approximation and relaxation techniques of AC power flow in radial systems are
} 
Following the zonal market design in Europe, we exclude transmission and distribution network constraints in the day-ahead stage, resulting in a single-node linear model. On the contrary, the real-time markets, one per scenario, include network constraints in both transmission and distribution systems. The real-time market-clearing problem is a conic program due to secondorder cone constraints within the power flow equations of distribution systems.

The interface optimizer is assumed to have enough data from the followers to be able to construct the bi-level model and anticipate their reactions. A practical implementation for market coordination may not require such a high degree of information sharing by using, e.g., a negotiation to determine the coordination variables. However, since we construct a benchmark tool to showcase the possibilities under such a framework, our aim is to examine the best case scenario, which is one in which all information is shared. This data availability assumption enables us to explore the maximum capability of the interface optimizer for improving the coordination between TSO and DSOs. One can expect that relaxing such an assumption may lower its capability, depending on the level of information asymmetry.

In order to calculate the real-time re-dispatch cost, topology information of both TSO and DSO networks is necessary. Both TSO and DSOs may be unwilling to share data about their network topology. It is assumed that this information is available to the interface optimizer, which is a reasonable assumption as we are examining the best possible outcome. Decentralized optimization such as the proposals in Caramanis et al. (2016) and Mohammadi et al. (2019) may in the future make it easier to coordinate in the real-time stage without sharing specific networkrelated proprietary information.

For the sake of simplicity, we exclude ramping constraints, energy storage, and other intertemporal couplings. Therefore, we consider a single hour only instead of a daily time horizon. Aligned with the European electricity markets where unit commitment constraints are internalized within the bids of market participants, the binary variables for the commitment status of conventional generators are neglected, such that both day-ahead and real-time market-clearing problems are convex. We also ignore the potential rebound effect of distributed energy resources (Greening et al., 2000), which is mostly the case for thermostatically controlled loads.

\section{Proposed stochastic bi-level model for the interface optimizer}

\subsection{Notation}

A full list of notation is listed below:

\section{Indices and sets:}

$\begin{array}{ll}d \in D & \text { Index for demands } \\ e \in E & \text { Index for distribution systems connected to the transmission grid } \\ g \in G & \text { Index for conventional generators } \\ n, m \in N & \text { Indices for nodes } \\ l \in L & \text { Index for lines. Note that } l=(n, m) \text { means line } l \text { connects nodes } n \text { and } m . \\ r \in R & \text { Index for weather-dependent renewable power units }\end{array}$

available in Taylor (2015) and Molzahn \& Hiskens (2019). In addition, Zohrizadeh et al. (2020) provide a detailed survey, showing the success of conic optimization for power system applications, in particular to solve optimal power flow problems in distribution systems. 


\section{Subsets and special labels:}

$D_{n}, G_{n}, R_{n}$ $D_{e}^{\mathrm{D}} \subset D$

$D^{\mathrm{T}} \subset D$

$G_{e}^{\mathrm{D}} \subset G$

$G^{\mathrm{T}} \subset G$

$L_{e}^{\mathrm{D}} \subset L$

$L^{\mathrm{T}} \subset L$

$N_{e}^{\mathrm{D}} \subset N$

$N^{\mathrm{T}} \subset N$

$\rightarrow n$

$n \rightarrow$

$n_{e}^{\mathrm{HV}} \in N^{\mathrm{T}}$

$n_{e}^{\mathrm{LV}} \in N_{e}^{\mathrm{D}}$

$l_{e}$

$l^{\prime}=(m, n)$

\section{Parameters:}

$\bar{P}_{g}, \underline{P}_{g}$

$\bar{P}_{d}, \underline{P}_{d}$

$\bar{Q}_{g}, \underline{Q}_{g}$

$\bar{Q}_{d}, \underline{Q}_{d}$

$B_{l}, R_{l}, X_{l}$

$S_{l}$

$\bar{V}_{n}, \underline{V}_{n}$

VOLL

$W_{r}^{\mathrm{DA}}$

$W_{r \omega}^{\mathrm{RT}}$

$\pi_{g}^{\mathrm{DA}}$
Subset of demands, generators, and renewable power units located at node $n$

Subset of demands connected to distribution system $e$

Subset of demands connected to the transmission grid

Subset of conventional generators connected to distribution system $e$

Subset of conventional generators connected to the transmission grid

Subset of lines in distribution system $e$

Subset of lines in the transmission grid

Subset of nodes in distribution system $e$

Subset of nodes in the transmission grid

Subset of lines ending at node $n$

Subset of lines originating at node $n$

Node at the high-voltage side of the interface between transmission and distribution system $e$

Node at the low-voltage side of the interface between transmission and distribution system $e$

Interface of distribution system $e$ connecting the high- and low-voltage side nodes

Line in the opposing direction of line $l$

Upper and lower active power limits for the output of generator $g$ [MW]

Upper and lower active power limits for the consumption of demand $d[\mathrm{MW}]$

Upper and lower reactive power limits for the output of generator $g$ [MVAr]

Upper and lower reactive power limits for the consumption of demand $d$ [MVAr]

Susceptance, resistance, and reactance of line $l$ [p.u.]

Capacity of line $l$ [MVA]

Upper and lower limits for the voltage magnitude of node $n$ [p.u.]

Value of lost load [\$/MWh]

Deterministic power forecast of renewable power unit $r$ in the day-ahead stage $[\mathrm{MW}]$

Actual power generation of renewable power unit $r$ realized in the real-time stage under scenario $\omega[\mathrm{MW}]$

Bid price of generator $g$ in the day-ahead stage [\$/MWh]

Bid price of demand $d$ in the day-ahead stage $[\$ / \mathrm{MWh}]$ 
$\pi^{\mathrm{R}}$

$\pi_{g}^{\downarrow}, \pi_{g}^{\uparrow}$

$\pi_{d}^{\downarrow}, \pi_{d}^{\uparrow}$

$\pi^{\downarrow \mathrm{R}}, \pi^{\uparrow \mathrm{R}}$

$\phi_{\omega}$

Bid price of renewable power units $[\$ / \mathrm{MWh}]$. It is assumed to be zero.

Regulating bid markup in the real-time stage for down- and up-regulation services provided by generator $g[\$ / \mathrm{MWh}]$

Regulating bid markup in the real-time stage for down- and up-regulation services provided by demand $d[\$ / \mathrm{MWh}]$

Bid price for down- and up-regulation services provided by the renewable power units in the real-time stage $[\$ / \mathrm{MWh}]$

Variables of the interface optimizer (coordination variables):

$\bar{f}_{e}, f_{e} \quad$ Upper and lower limits on import/export of apparent power at the interface of distribution system $e$ with the transmission grid [MVA]

$\pi_{e}^{\mathrm{IO}, \mathrm{DA}} \quad$ Day-ahead price at the interface of distribution system $e$ with the transmission grid $[\$ / \mathrm{MWh}]$

$\pi_{e}^{\downarrow I O}, \pi_{e}^{\uparrow \mathrm{IO}} \quad$ Regulating bid markup for down- and up-regulation services in the real-time stage at the interface of distribution system $e$ with the transmission grid $[\$ / \mathrm{MWh}]$

Variables at the day-ahead stage (here-and-now decisions):

$\widetilde{p}_{g}^{\mathrm{DA}}, \widetilde{p}_{d}^{\mathrm{DA}}$

Active power dispatch of DSO-level generator $g$ and DSO-level demand $d$ in the local DSO market $[\mathrm{MW}$. These two values are outcomes of the prequalification performed by the DSO, and are treated as a cap on the quantity bid of the generator/demand to the wholesale day-ahead market.

$\widehat{p}_{g}^{\mathrm{DA}}, \widehat{p}_{d}^{\mathrm{DA}} \quad$ Active power dispatch of generator $g$ and demand $d$ in the day-ahead market [MW]

$s^{\mathrm{DA}} \quad$ Total active power load not supplied in the day-ahead market [MW]

$w_{r}^{\mathrm{DA}} \quad$ Active power dispatch of renewable power unit $r$ in the day-ahead market $[\mathrm{MW}]$

Variables at the real-time stage under scenario $\omega$ (wait-and-see decisions):

$p_{g \omega}^{\downarrow}, p_{g \omega}^{\uparrow}$

$p_{d \omega}^{\downarrow}, p_{d \omega}^{\uparrow}$

$p_{g \omega}^{\mathrm{RT}}, q_{g \omega}^{\mathrm{RT}}$

$p_{d \omega}^{\mathrm{RT}}, q_{d \omega}^{\mathrm{RT}}$
Active power down- and up-regulation services provided by generator $g$ in the real-time stage under scenario $\omega[\mathrm{MW}]$

Active power down- and up-regulation services provided by demand $d$ in the real-time stage under scenario $\omega[\mathrm{MW}]$

Final active $[\mathrm{MW}]$ and reactive [MVAr] power production levels of generator $g$ in the real-time stage under scenario $\omega$. By final, we refer to the day-ahead dispatch plus the regulation service provided in the real-time.

Final active $[\mathrm{MW}]$ and reactive [MVAr] power consumption levels of demand $d$ in the real-time stage under scenario $\omega$. By final, we refer to the day-ahead dispatch plus the regulation service provided in the real-time. 


$\begin{array}{ll}p_{l \omega}^{\mathrm{RT}}, q_{l \omega}^{\mathrm{RT}} & \begin{array}{l}\text { Final active }[\mathrm{MW}] \text { and reactive [MVAr] power flow across line } l \text { in the real-time } \\ \text { stage under scenario } \omega \text {. By final, we refer to the day-ahead dispatch plus the }\end{array} \\ & \text { regulation service provided in the real-time. } \\ s_{n \omega}^{\mathrm{RT}} & \text { Total active power load curtailed at node } n \text { in the real-time stage under sce- } \\ & \text { nario } \omega \text { [MW] } \\ s_{n \omega}^{\mathrm{q}, \mathrm{RT}} & \text { Total reactive power load curtailed at node } n \text { in the real-time stage under } \\ & \text { scenario } \omega[\mathrm{MVAr}] \\ \theta_{n \omega}^{\mathrm{RT}} & \text { Voltage angle at the TSO-level node } n \text { in the real-time stage under scenario } \omega \\ w_{r \omega}^{\downarrow}, w_{r \omega}^{\uparrow} & \text { [rad] } \\ & \text { Active power down- and up-regulation services provided by renewable power } \\ w_{r \omega}^{\mathrm{RT}} & \text { unit } r \text { in the real-time stage under scenario } \omega \text { [MW] } \\ & \text { Final active power production of renewable power unit } r \text { in the real-time stage } \\ & \text { under scenario } \omega[\text { MW]. By final, we refer to the day-ahead dispatch plus the } \\ & \text { regulation service provided in the real-time. } \\ v_{n \omega}^{\mathrm{RT}} & \text { Squared voltage magnitude at the DSO-level node } n \text { in the real-time stage } \\ & \text { under scenario } \omega \text { [p.u.] } \\ \varphi_{l \omega}^{\mathrm{RT}} & \text { Squared current magnitude flow in the DSO-level line } l \text { in the real-time stage } \\ & \text { under scenario } \omega[\text { p.u.] }\end{array}$

\subsection{Stochastic bi-level model}

As mentioned earlier, the single leader in the proposed stochastic bi-level model is the interface optimizer, whose goal is to maximize the expected social welfare in entire system, including both transmission and distributions systems, and accounting for both day-ahead and real-time trading stages. The three sequential followers are the local DSO markets (one per distribution system), day-ahead market, and real-time markets (one per scenario). A schematic illustration of the proposed bi-level structure is provided in Fig. 2a. The interface optimizer as the leader determines the optimal values of coordination variables at all interfaces, collected in the variable set $\Xi^{\mathrm{IO}}=\left\{\bar{f}_{e}, \underline{f}_{e}, \pi_{e}^{\mathrm{IO}, \mathrm{DA}}, \pi_{e}^{\uparrow \mathrm{IO}}, \pi_{e}^{\downarrow \mathrm{IO}} \forall e\right\}$. For given values of these coordination variables, the DSO markets, one for each distribution system $e$, perform a pre-qualification to find the optimal restriction to impose to local generators and demands on how much they are allowed to participate in the wholesale day-ahead market. This way, each DSO ensures the satisfaction of its local AC power flow constraints, modeled by a conic program. The DSO restrictions are imposed by caps $\widetilde{p}_{g}^{\mathrm{DA}}$ and $\widetilde{p}_{d}^{\mathrm{DA}}$. For given values of these caps, the day-ahead market is cleared using a linear program. The objective of the day-ahead market clearing is to maximize the social welfare at the day-ahead stage, i.e., $\mathcal{S} \mathcal{W}^{\mathrm{DA}}$. For given outcomes of the day-ahead market, i.e., dispatch of generators $\widehat{p}_{g}^{\mathrm{DA}}$ and dispatch of demands $\widehat{p}_{d}^{\mathrm{DA}}$, the real-time market, under each scenario, is cleared to adjust the renewable power imbalances, i.e., the deviation of actual renewable power with respect to its day-ahead dispatch. Since demands are given at this stage, the objective of the real-time market clearing under each scenario $\omega$ reduces to the minimization of social cost under that scenario, i.e., $\Delta$ Cost $_{\omega}^{\mathrm{RT}}$. The real-time market embodies both transmission and distribution levels, and therefore its clearing problem is a conic program. The optimal social welfare in the day-ahead stage and the social cost in the real-time stage, i.e., $\mathcal{S} \mathcal{W}^{\mathrm{DA}}$ and $\Delta \operatorname{Cost}_{\omega}^{\mathrm{RT}}$, construct the interface optimizer's objective function, and this way, the followers impact the leader's decisions. The mathematical formulation of the proposed bi-level model writes as 


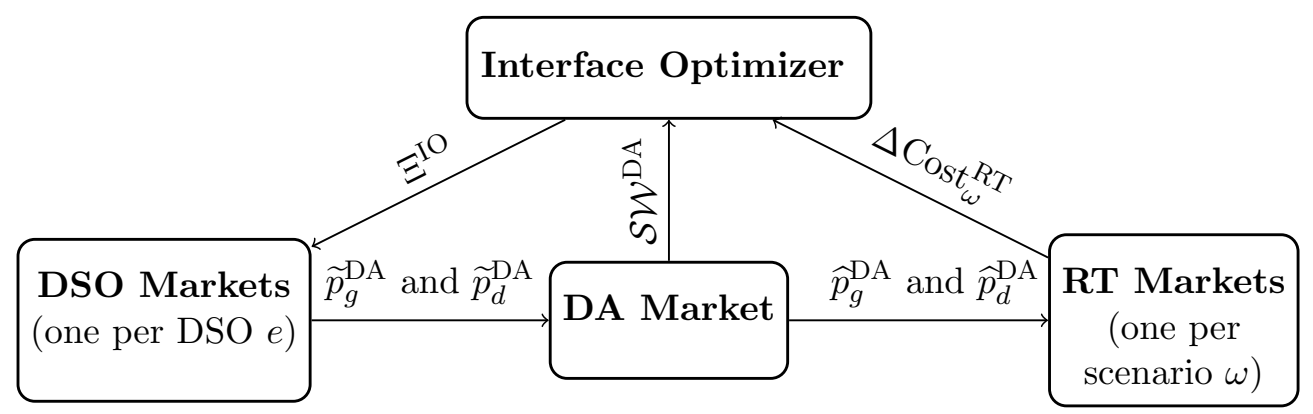

(a) Structure of the proposed stochastic bi-level problem (1). This problem will be equivalently reduced to the bi-level structure in the lower plot (Fig. 2b). DA: day-ahead; RT: real-time.

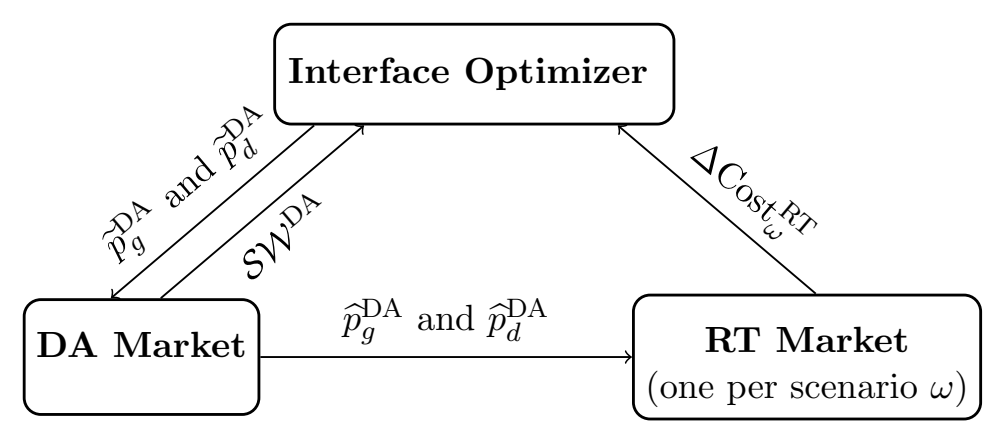

(b) Structure of the reduced bi-level problem (2). According to Proposition 1 , this problem is equivalent to the original bi-level problem (1) illustrated in Fig. 2a, assuming information symmetry between the interface optimizer and the DSOs. DA: day-ahead; RT: real-time.

Figure 2: Structure of two equivalent bi-level problems.

$$
\max _{\Xi^{\mathrm{IO}}} \mathcal{S} \mathcal{W}^{\mathrm{DA}}-\sum_{\omega} \phi_{\omega} \Delta \operatorname{Cost}_{\omega}^{\mathrm{RT}}
$$

subject to:

$\widetilde{p}_{g}^{\mathrm{DA}}, \widetilde{p}_{d}^{\mathrm{DA}} \in \arg \left\{(\mathrm{C} .1)\right.$ for given $\left.\Xi^{\mathrm{IO}}\right\}, \forall e$,

$\mathcal{S} \mathcal{W}^{\mathrm{DA}}, \widehat{p}_{g}^{\mathrm{DA}}, \widehat{p}_{d}^{\mathrm{DA}} \in \arg \left\{(3)\right.$ for given $\left.\widetilde{p}_{g}^{\mathrm{DA}}, \widetilde{p}_{d}^{\mathrm{DA}}\right\}$,

$\Delta \operatorname{Cost}_{\omega}^{\mathrm{RT}} \in \arg \left\{(4)\right.$ for given $\left.\widehat{p}_{g}^{\mathrm{DA}}, \widehat{p}_{d}^{\mathrm{DA}}\right\}, \forall \omega$.

The interface optimizer maximizes the upper-level objective function (1a), including the dayahead social welfare minus the expected social cost in the real-time stage. This objective function is constrained by three lower-level problems (1b), (1c) and (1d), referring to the DSO markets, dayahead market, and real-time markets, respectively. These constraints represent three sequential followers illustrated in Fig. 2a. Each of these lower-level problems (1b)-(1d) is itself an optimization problem. This makes the whole problem (1) a bi-level model, i.e., an optimization problem constrained by other optimization problems. This will be eventually recast as a complementarity 
model. The lower-level problem (1b) embodies the optimization problem of each DSO market, whose full mathematical model is given by problem (C.1) in the online appendix (Hermann et al., 2021). The reason for not including it in the paper is that we will demonstrate soon that the bi-level model (1) can be equivalently reduced to another bi-level model excluding (1b). The lower-level problems (1c) and (1d) include day-ahead and real-time market-clearing problems, whose mathematical formulations will be given later as optimization problems (3) and (4).

In general, bi-level problem (1) is computationally hard to solve, especially because we model the DSO markets, one per DSO, and real-time markets, one per scenario, in the lower-level problems. Recall that both DSO and real-time market-clearing problems are conic programs.

For the sake of simplicity, we assume that the interface optimizer has complete knowledge about DSO markets. This means that the interface optimizer has access to all technical data of distribution systems. In addition, the interface optimizer and all DSOs have the same beliefs about the probability distribution of uncertainty and the set of scenarios generated. This assumption is so-called information symmetry between the interface optimizer and DSOs. As mentioned earlier in Section 1, we see our proposed method as a tool to show the maximum potential for TSO-DSO coordination by optimizing the interface characteristics at the day-ahead stage, and the assumption of information symmetry is fully aligned with our purpose. By relaxing this assumption, one can hypothesize that the value of the proposed TSO-DSO coordination will decrease, depending on the magnitude of information asymmetry. In addition, under the assumption of information symmetry, we are able to simplify the original bi-level problem (1) by excluding lower-level problem (1b), as described by the following proposition:

Proposition 1: Under the assumption of information symmetry between the interface optimizer and DSOs, the reduced bi-level problem (2) is equivalent to the original bi-level model (1). This implies that a solution to (2) is a solution to (1), and vice versa.

Proof: In the original bi-level model (1), all lower-level problems (1b), (1c), and (1d) are convex, and we can derive their optimality KKT conditions. These conditions are provided in the online appendix (Hermann et al., 2021). The KKT conditions associated with lower-level problem (1b) are redundant. The exact same conditions are contained in the KKT conditions associated with lower-level problems (1c) and (1d). Therefore, the KKT conditions associated with lower-level problem (1b) can be removed.

The schematic illustration of this reduced bi-level problem is given in Fig. 2b. This reduced bi-level problem writes as

$$
\begin{aligned}
& \max _{\widetilde{p}_{g}^{\mathrm{DA}}, \widetilde{p}_{d}^{\mathrm{DA}}} \mathcal{S} \mathcal{W}^{\mathrm{DA}}-\sum_{\omega} \phi_{\omega} \Delta \operatorname{Cost}_{\omega}^{\mathrm{RT}} \\
& \text { subject to: } \\
& 0 \leq \widetilde{p}_{g}^{\mathrm{DA}} \leq \bar{P}_{g} \quad \forall e, g \in G_{e}^{\mathrm{D}} \\
& 0 \leq \widetilde{p}_{d}^{\mathrm{DA}} \leq \bar{P}_{d} \forall e, d \in D_{e}^{\mathrm{D}} \\
& \mathcal{S} \mathcal{W}^{\mathrm{DA}}, \widehat{p}_{g}^{\mathrm{DA}}, \widehat{p}_{d}^{\mathrm{DA}} \in \arg \left\{(3) \text { for given } \widetilde{p}_{g}^{\mathrm{DA}}, \widetilde{p}_{d}^{\mathrm{DA}}\right\}, \\
& \Delta \operatorname{Cost}_{\omega}^{\mathrm{RT}} \in \arg \left\{(4) \text { for given } \widehat{p}_{g}^{\mathrm{DA}}, \widehat{p}_{d}^{\mathrm{DA}}\right\}, \forall \omega .
\end{aligned}
$$


In the reduced bi-level problem (2), the interface optimizer directly imposes the caps on quantity bids of local distributed energy resources through upper-level constraints (2b) and (2c). The upperlevel objective function (2a) and lower-level problems (2d) and (2e) are the same as those in the original bi-level problem (1).

We now present the mathematical formulation of lower-level problems corresponding to the day-ahead and real-time market clearing. The objective of the day-ahead market-clearing operator is the social welfare maximization. Following the European zonal day-ahead markets, this market is a pool, including bids of all agents in TSO and DSO levels, without modeling network constraints. However, the network constraints will be considered later in the real-time stage. The day-ahead market-clearing problem writes as

$$
\max _{\widehat{p}_{g}^{\mathrm{DA}}, \widehat{p}_{d}^{\mathrm{AA}}, s^{\mathrm{DA}}, w_{r}^{\mathrm{DA}}} \mathcal{S} \mathcal{W}^{\mathrm{DA}}=\sum_{d \in D} \pi_{d}^{\mathrm{DA}} \widehat{p}_{d}^{\mathrm{DA}}-\sum_{g \in G} \pi_{g}^{\mathrm{DA}} \widehat{p}_{g}^{\mathrm{DA}}-\pi^{\mathrm{R}} \sum_{r} w_{r}^{\mathrm{DA}}-V O L L s^{\mathrm{DA}}
$$

subject to:

$$
\begin{aligned}
& \sum_{g \in G} \widehat{p}_{g}^{\mathrm{DA}}-\sum_{d \in D} \widehat{p}_{d}^{\mathrm{DA}}+\sum_{r} w_{r}^{\mathrm{DA}}+s^{\mathrm{DA}}=0 \\
& \underline{P}_{g} \leq \widehat{p}_{g}^{\mathrm{DA}} \leq \widehat{p}_{g}^{\mathrm{DA}}, \quad \forall e, g \in G_{e}^{\mathrm{D}} \\
& \underline{P}_{g} \leq \widehat{p}_{g}^{\mathrm{DA}} \leq \bar{P}_{g}, \quad \forall g \in G^{\mathrm{T}} \\
& \underline{P}_{d} \leq \widehat{p}_{d}^{\mathrm{DA}} \leq \widehat{p}_{d}^{\mathrm{DA}}, \quad \forall e, d \in D_{e}^{\mathrm{D}} \\
& \underline{P}_{d} \leq \widehat{p}_{d}^{\mathrm{DA}} \leq \bar{P}_{d}, \quad \forall d \in D^{\mathrm{T}} \\
& 0 \leq w_{r}^{\mathrm{DA}} \leq W_{r}^{\mathrm{DA}}, \forall r \in R \\
& 0 \leq s^{\mathrm{DA}} \leq \sum_{d} \widehat{p}_{d}^{\mathrm{DA}},
\end{aligned}
$$

which is a deterministic linear program. The caps on quantity bids of DSO-level generators and demands, i.e., $\widetilde{p}_{g}^{\mathrm{DA}}$ and $\widetilde{p}_{d}^{\mathrm{DA}}$, are treated as parameters in (3), while they are decision variables for the interface optimizer in (2). The objective function (3a) maximizes the social welfare, i.e., utility of demands minus the production cost of conventional generators, renewable power units, and the load curtailment cost. Constraint (3b) enforces the global power balance, considering all generators and demands in both TSO and DSO levels. Constraints (3c)-(3h) limit the power quantities dispatched. It is worth mentioning that the caps $\widetilde{p}_{g}^{\mathrm{DA}}$ and $\widetilde{p}_{d}^{\mathrm{DA}}$ are only enforced for DSOlevel generators and demands in (3c) and (3e). In contrast, the dispatch of TSO-level generators and demands is restricted by their real capacity in (3d) and (3f).

Under each scenario $\omega$, the real-time market re-dispatches the generators and demands in both TSO and DSO levels considering the full nodal power flow. For the radial distribution networks, a convex relaxation of AC power flow (Farivar \& Low, 2013) is employed, yielding an $\mathrm{SOCP}^{13}$. This model allows for taking into account the reactive power flows over lines, active and reactive power

\footnotetext{
${ }^{13}$ In general, the conic relaxation of AC optimal power flow problem in radial distribution systems is tight, but not necessarily exact. An exact conic relaxation can be obtained if some sufficient conditions for relaxation are added to the problem. However, these conditions may shrink the feasible space of the AC optimal power problem, resulting in a sub-optimal but always-feasible solution. It is still a discussion point in the research community how mild these sufficient conditions are. See further discussions in Low (2014); Nick et al. (2018), and Bobo et al. (2021). We do not add such sufficient conditions in this paper.
} 
losses, and nodal voltage magnitudes in distribution networks. In contrast, for the transmission network, a linearized approximate power flow model is used, discarding the reactive power flows, active and reactive power losses, as well as nodal voltage magnitudes. Therefore, the real-time market-clearing problem under scenario $\omega$ is an SOCP, and writes as

$$
\begin{aligned}
& \min _{\Xi_{\omega}^{\mathrm{RT}}} \Delta \operatorname{Cost}_{\omega}^{\mathrm{RT}}=\operatorname{VOLL} \sum_{n \in N} s_{n \omega}^{\mathrm{RT}}+\sum_{g \in G}\left(\pi_{g}^{\mathrm{DA}}\left(p_{g \omega}^{\mathrm{RT}}-\widehat{p}_{g}^{\mathrm{DA}}\right)+\pi_{g}^{\uparrow} p_{g \omega}^{\uparrow}+\pi_{g}^{\downarrow} p_{g \omega}^{\downarrow}\right) \\
& +\sum_{d \in D}\left(\pi_{d}^{\mathrm{DA}}\left(\widehat{p}_{d}^{\mathrm{DA}}-p_{d \omega}^{\mathrm{RT}}\right)+\pi_{d}^{\uparrow} p_{d \omega}^{\uparrow}+\pi_{d}^{\downarrow} p_{d \omega}^{\downarrow}\right) \\
& +\sum_{r}\left(\pi^{\mathrm{R}}\left(w_{r \omega}^{\mathrm{RT}}-w_{r}^{\mathrm{DA}}\right)+\pi^{\uparrow \mathrm{R}} w_{r \omega}^{\uparrow}+\pi^{\downarrow \mathrm{R}} w_{r \omega}^{\downarrow}\right)
\end{aligned}
$$

subject to:

$$
\begin{aligned}
& p_{l \omega}^{\mathrm{RT}}=B_{l}\left(\theta_{n \omega}-\theta_{m \omega}\right), \quad \forall l \in L^{\mathrm{T}} \\
& p_{l \omega}^{\mathrm{RT}} \leq S_{l}, \forall l \in L^{\mathrm{T}} \\
& p_{g \omega}^{\mathrm{RT}}=\widehat{p}_{g}^{\mathrm{DA}}+p_{g \omega}^{\uparrow}-p_{g \omega}^{\downarrow}, \quad \forall g \in G \\
& w_{r \omega}^{\mathrm{RT}}=w_{r}^{\mathrm{DA}}+w_{r \omega}^{\uparrow}-w_{r \omega}^{\downarrow}, \forall r \in R \\
& p_{d \omega}^{\mathrm{RT}}=\widehat{p}_{d}^{\mathrm{DA}}-p_{d \omega}^{\uparrow}+p_{d \omega}^{\downarrow}, \quad \forall d \in D \\
& \sum_{g \in G_{n}} p_{g \omega}^{\mathrm{RT}}-\sum_{d \in D_{n}} p_{d \omega}^{\mathrm{RT}}+\sum_{r \in R_{n}} w_{r \omega}^{\mathrm{RT}}+s_{n \omega}^{\mathrm{RT}}=\sum_{l \in n \rightarrow} p_{l \omega}^{\mathrm{RT}}-\sum_{l \in \rightarrow n} p_{l \omega}^{\mathrm{RT}}, \forall n \in N \\
& \sum_{g \in G_{n}} q_{g \omega}^{\mathrm{RT}}-\sum_{d \in D_{n}} q_{d \omega}^{\mathrm{RT}}+s_{n \omega}^{\mathrm{q}, \mathrm{RT}}=\sum_{l \in n \rightarrow} q_{l \omega}^{\mathrm{RT}}-\sum_{l \in \rightarrow n} q_{l \omega}^{\mathrm{RT}}, \forall e, n \in N_{e}^{\mathrm{D}} \\
& p_{l \omega}^{\mathrm{RT}^{2}}+q_{l \omega}^{\mathrm{RT}^{2}} \leq \varphi_{l \omega}^{\mathrm{RT}} v_{n \omega}^{\mathrm{RT}}, \forall e, l \in\left(L_{e}^{\mathrm{D}} \cup l_{e}\right) \\
& p_{l \omega}^{\mathrm{RT}^{2}}+q_{l \omega}^{\mathrm{RT}^{2}} \leq S_{l}^{2}, \forall e, l \in\left(L_{e}^{\mathrm{D}} \cup l_{e}\right) \\
& p_{l \omega}^{\mathrm{RT}}+p_{l^{\prime} \omega}^{\mathrm{RT}}=R_{l} \varphi_{l \omega}^{\mathrm{RT}}, \forall e, l \in\left(L_{e}^{\mathrm{D}} \cup l_{e}\right) \\
& q_{l \omega}^{\mathrm{RT}}+q_{l^{\prime} \omega}^{\mathrm{RT}}=X_{l} \varphi_{l \omega}^{\mathrm{RT}}, \forall e, l \in\left(L_{e}^{\mathrm{D}} \cup l_{e}\right) \\
& v_{m \omega}^{\mathrm{RT}}=v_{n \omega}^{\mathrm{RT}}-2\left(R_{l} p_{l \omega}^{\mathrm{RT}}+X_{l} q_{l \omega}^{\mathrm{RT}}\right)+\left(R_{l}^{2}+X_{l}^{2}\right) \varphi_{l \omega}^{\mathrm{RT}}, \forall e, l \in\left(L_{e}^{\mathrm{D}} \cup l_{e}\right) \\
& \underline{V}_{n}^{2} \leq v_{n \omega}^{\mathrm{RT}} \leq \bar{V}_{n}^{2}, \quad \forall e, n \in\left(N_{e}^{\mathrm{D}} \cup n_{e}^{\mathrm{HV}}\right) \\
& 0 \leq w_{r \omega}^{\mathrm{RT}} \leq W_{r \omega}^{\mathrm{RT}}, \quad \forall r \in R \\
& \underline{P}_{g} \leq p_{g \omega}^{\mathrm{RT}} \leq \bar{P}_{g}, \quad \forall g \in G \\
& \underline{P}_{d} \leq p_{d \omega}^{\mathrm{RT}} \leq \bar{P}_{d}, \quad \forall d \in D \\
& \underline{Q}_{g} \leq q_{g \omega}^{\mathrm{RT}} \leq \bar{Q}_{g}, \quad \forall g \in G_{e}^{\mathrm{D}} \\
& \underline{Q}_{d} \leq q_{d \omega}^{\mathrm{RT}} \leq \bar{Q}_{d}, \quad \forall d \in D_{e}^{\mathrm{D}} \\
& 0 \leq s_{n \omega}^{\mathrm{RT}} \leq \sum_{d \in D_{n}} p_{d \omega}^{\mathrm{RT}}, \forall n \in N \\
& p_{g \omega}^{\uparrow} \geq 0, \forall g, \quad p_{g \omega}^{\downarrow} \geq 0, \forall g \\
& p_{d \omega}^{\uparrow} \geq 0, \forall d, \quad p_{d \omega}^{\downarrow} \geq 0, \forall d
\end{aligned}
$$




$$
w_{r \omega}^{\uparrow} \geq 0, \forall r, \quad w_{r \omega}^{\downarrow} \geq 0, \forall r
$$

where variable set $\Xi_{\omega}^{\mathrm{RT}}$ includes $p_{g \omega}^{\mathrm{RT}}, p_{d \omega}^{\mathrm{RT}}, w_{r \omega}^{\mathrm{RT}}, p_{g \omega}^{\uparrow}, p_{g \omega}^{\downarrow}, p_{d \omega}^{\uparrow}, p_{d \omega}^{\downarrow}, w_{r \omega}^{\uparrow}, w_{r \omega}^{\downarrow}, s_{n \omega}^{\mathrm{RT}}, s_{n \omega}^{\mathrm{q}, \mathrm{RT}}, q_{g \omega}^{\mathrm{RT}}, q_{d \omega}^{\mathrm{RT}}$, $p_{l \omega}^{\mathrm{RT}}, q_{l \omega}^{\mathrm{RT}}, \theta_{n \omega}, \varphi_{l \omega}^{\mathrm{RT}}$, and $v_{n \omega}^{\mathrm{RT}}$. The objective function (4a) minimizes the social cost, comprising of the cost of curtailed load, and the re-dispatch cost of conventional generators, demands, and renewable power units. Note that a premium on up- and down-regulation costs is considered. Constraint (4b) uses the lossless linear representation of power flow to determine the active power flow across lines in the TSO level, whereas (4c) imposes the transmission line capacity limits. Constraints (4d) and (4e) determine the final active power production of each conventional generator and renewable power unit, and (4f) computes the final consumption of each demand. By final, we refer to the day-ahead dispatch plus the real-time regulation. The nodal active power balance in both TSO and DSO levels is enforced by $(4 \mathrm{~g})$, whereas the nodal reactive power balance in the DSO level is imposed by $(4 \mathrm{~h})$. The second-order cone constraints $(4 \mathrm{i})$ and $(4 \mathrm{j})$ enforce the capacity of distribution lines. Note that (4i) is a quadratic equality and therefore a non-convex constraint in the original $\mathrm{AC}$ power flow model, but it is relaxed here to achieve convexity. In an exact relaxation, this constraint is supposed to be binding. Active and reactive power losses in distribution network are modeled in (4k) and (4l), whereas nodal voltage magnitudes are calculated by $(4 \mathrm{~m})$. Constraints $(4 \mathrm{n})$ through $(4 \mathrm{t})$ impose lower and upper bounds for different variables. Finally, $(4 \mathrm{u})-(4 \mathrm{w})$ declare non-negativity conditions.

Since (4) solves a centralized optimal power flow problem with all nodes in both transmission and distribution networks, it is not practical to implement this problem in reality. Several other works have shown how this problem can be decomposed into separate problems, which are more manageable for large cases (Caramanis et al., 2016; Mohammadi et al., 2019). The implementation and discussion of such a distributed problem is however considered outside the scope of this paper.

\section{Solution strategy and benchmarks}

\subsection{Benders' decomposition}

The most common way to solve bi-level problem (2) is to replace lower-level problems (2d) and (2e) by their optimality KKT conditions. The resulting single-level model will be a mathematical program with equilibrium constraints (Luo et al., 1996). However, it is a computationally expensive solution, especially for large case studies with many scenarios. This motivates us to use a decomposition technique and split (2) to a set of smaller problems.

We use a Benders' decomposition method (Benders, 1962) by choosing the day-ahead dispatch decisions, i.e., $\widehat{p}_{g}^{\mathrm{DA}}, \widehat{p}_{d}^{\mathrm{DA}}$ and $w_{r}^{\mathrm{DA}}$, as complicating (coupling) variables (Conejo et al., 2006). In particular, we use a multi-cut version of Benders' decomposition, which accelerates the convergence (Birge \& Louveaux, 1988). By fixing these variables, the original bi-level problem (2) decomposes into a master problem for the interface optimizer and a set of sub-problems for real-time re-dispatch under each scenario. The master problem at iteration $(i)$ reads as

$$
\max _{\Xi^{\mathrm{MP}^{(i)}}} \mathcal{S} \mathcal{W}^{\mathrm{DA}^{(i)}}-\sum_{\omega} \phi_{\omega} \psi_{\omega}^{(i)}
$$

subject to:

$$
\begin{aligned}
& (2 \mathrm{~b})-(2 \mathrm{c}) \\
& \mathcal{S} \mathcal{W}^{\mathrm{DA}^{(i)}} \in(3)
\end{aligned}
$$




$$
\begin{aligned}
& \psi_{\omega}^{(i)} \geq \psi^{\mathrm{min}}, \quad \forall \omega \in \Omega \\
& \psi_{\omega}^{(i)} \geq \Delta \operatorname{Cost}_{\omega}^{\mathrm{RT}}{ }^{(m)}+\sum_{g \in G} \alpha_{g \omega}^{(m)}\left(\widehat{p}_{g}^{\mathrm{DA}^{(i)}}-\widehat{p}_{g}^{\mathrm{DA}}{ }^{(m)}\right)+\sum_{d \in D} \alpha_{d \omega}^{(m)}\left(\widehat{p}_{d}^{\mathrm{DA}^{(i)}}-\widehat{p}_{g}^{\mathrm{DA}^{(m)}}\right) \\
& +\sum_{r \in R} \alpha_{r \omega}^{(m)}\left(w_{r}^{\mathrm{DA}^{(i)}}-w_{r}^{\mathrm{DA}^{(m)}}\right), \quad \forall m \in\{1, \ldots, i-1\}, \omega,
\end{aligned}
$$

where variable set $\Xi^{\mathrm{MP}^{(i)}}$ includes $\widehat{p}_{g}^{\mathrm{DA}^{(i)}}, \widehat{p}_{d}^{\mathrm{DA}^{(i)}}, s^{\mathrm{DA}^{(i)}}, w_{r}^{\mathrm{DA}^{(i)}}, \widetilde{p}_{g}^{\mathrm{DA}^{(i)}}, \widetilde{p}_{d}^{\mathrm{DA}^{(i)}}$ and $\psi_{\omega}^{(i)}$. The master problem (5) is still a bi-level problem, whose lower-level problem is (5c). Similar to the objective function (2a) of the non-decomposed bi-level problem, the objective function (5a) maximizes the day-ahead social welfare minus the expected re-dispatch cost in the real-time operation. The latter is represented by the auxiliary variable $\psi_{\omega}^{(i)}$. This variable is constrained by a lower bound in (5d) to avoid unbounded solution in the initial iteration, and by multiple cuts in (5e), one per scenario. Note that $(m)$ is an index for previous iterations, and all symbols with superscript $(m)$ are parameters, whose values come from the solution of the master and sub-problems in the previous iterations. In particular, $\alpha_{g \omega}^{(m)}, \alpha_{d \omega}^{(m)}$, and $\alpha_{r \omega}^{(m)}$ are dual variables in the sub-problems, and their values are obtained from sub-problems in the previous iterations. Note also that (5e) includes optimality cuts only, and there is no need for the inclusion of the feasibility cuts. The reason for this is that the sub-problems are always feasible due to the availability of extreme corrective actions, i.e., wind spillage and load curtailment.

This master problem as a bi-level program can be solved by replacing the linear problem (3) in (5c) by its KKT optimality conditions, as given in the online appendix (Hermann et al., 2021). After linearizing the complementarity conditions using a Big-M method ${ }^{14}$ (Fortuny-Amat \& McCarl, 1981), which requires auxiliary binary variables, the master problem (5) boils down to a mixed-integer linear problem.

One computational observation is that since we generate multiple cuts, one per scenario, at each iteration, the size of master problem might be quickly increased (You \& Grossmann, 2013). In that case, the computational issues may occur. Therefore, it is important to get convergence in a few number of iterations. If it is not the case, then it is likely more desirable to use the regular version of Benders' decomposition, which generates a single cut at each iteration. Another potential alternative is to use an adaptive approach for aggregation of multicuts as proposed in Trukhanov et al. (2010) and Skar et al. (2014). A comprehensive review on Benders' decomposition and its different variants is available in Rahmaniani et al. (2017).

It is worth noting that the proposed Benders' decomposition algorithm would still work if the assumption of information symmetry is relaxed. However, Proposition 1 in Section 3 will no longer hold, meaning that the master problem (5) will include not only the lower-level problem for the day-ahead market clearing, but also the lower-level problem for each DSO's optimization problem. This will further complicate the master problem.

Recall that the master problem determines the dispatch of conventional generators, renewable power units, and demands based on the available single-point deterministic renewable power forecast in the day-ahead stage. However, the realization of renewable power generation might be

\footnotetext{
${ }^{14}$ In Big-M method proposed in Fortuny-Amat \& McCarl (1981), the choice of proper values for big constants could be challenging, such that a wrong choice may cause sub-optimality or numerical ill-conditioning. The interested reader is referred to Pineda \& Morales (2019) and Kleinert et al. (2020) for further details about the potential techniques for proper selection of these values.
} 
different than the day-ahead forecast. Therefore, given the day-ahead dispatch values obtained from the master problem (5), each sub-problem takes into account a distinct scenario for renewable power generation, and determines the magnitude of renewable power imbalance between the day-ahead dispatch of renewable power units and their realized production in the underlying scenario. This sub-problem activates the flexible resources, e.g., flexible generators and controllable demands, in both TSO and DSO levels to provide regulation services and offset the renewable power imbalance under that scenario. The goal of this sub-problem is to minimize the social cost incurred by the regulation service provision. This sub-problem for scenario $\omega$ at iteration $(i)$ reads as

$$
\min _{\Xi_{\omega} \mathrm{RT}^{(i)}} \Delta \operatorname{Cost}_{\omega}^{\mathrm{RT}}{ }^{(i)}
$$

subject to:

$$
\begin{aligned}
& \Delta \operatorname{Cost}_{\omega}^{\mathrm{RT}}{ }^{(i)}, \Xi_{\omega}^{\mathrm{RT}(\mathrm{i})} \in(4) \\
& \widehat{p}_{g}^{\mathrm{DA}^{(i)}}=\widehat{p}_{g}^{\mathrm{DA}^{\mathrm{DAxed}}{ }^{(i)}}, \\
& \widehat{p}_{d}^{\mathrm{DA}^{(i)}}=\widehat{p}_{d}^{\mathrm{DA}, \mathrm{fixed}^{(i)}}, \\
& w_{r}^{\mathrm{DA}^{(i)}}=w_{r}^{\mathrm{DA}, \mathrm{fixed}^{(i)}},
\end{aligned}
$$

Each sub-problem (6) at iteration $(i)$ is a bi-level problem, whose lower level is SOCP (4) in (6b). The dual variables $\alpha_{g \omega}^{(i)}, \alpha_{d \omega}^{(i)}$, and $\alpha_{r \omega}^{(i)}$ are defined for every conventional generator, demand, and renewable power unit, which are used to construct cuts (5e) in the master problem. One important observation is that the objective function (6a) is the same as in (4) within (6b). Therefore, the bi-level structure of (6) can be eliminated, and each sub-problem reduces to a single-stage SOCP as

$$
\min _{\Xi_{\omega}^{\mathrm{RT}^{(i)}}} \Delta \operatorname{Cost}_{\omega}^{\mathrm{RT}}{ }^{(i)} \text { subject to: }(4 \mathrm{~b})-(4 \mathrm{w}) \text { and }(6 \mathrm{c})-(6 \mathrm{e}) .
$$

The iterative Benders' decomposition algorithm finds an optimal solution for the bi-level problem (2) with a level of accuracy $\epsilon$ if a lower bound $\mathrm{LB}^{(i)}=-\sum_{\omega} \phi_{\omega} \psi_{\omega}^{(i)}$ and an upper bound $\mathrm{UB}^{(i)}=-\sum_{\omega} \phi_{\omega} \Delta \operatorname{Cost}_{\omega}^{\mathrm{RT}^{(i)}}$ converge to within a predefined discrepancy $\epsilon \geq \mathrm{UB}^{(i)}-\mathrm{LB}^{(i)}$. This convergence is guaranteed by convexity of sub-problems (7). As an alternative, one can include the original quadratic equality constraints of $\mathrm{AC}$ power flow equations instead of their relaxed variant, i.e., (4i). However, this makes the sub-problems non-convex, requiring a non-linear non-convex generalized Benders' decomposition algorithm (Frank \& Rebennack, 2015; Li et al., 2011).

\subsection{Two benchmark models}

To assess the performance of the proposed coordination mechanism based on optimizing the interface characteristics, we define here two benchmarks, one is an ideal benchmark providing an upper bound for the expected social welfare of the whole system, and the other one provides a lower bound. 


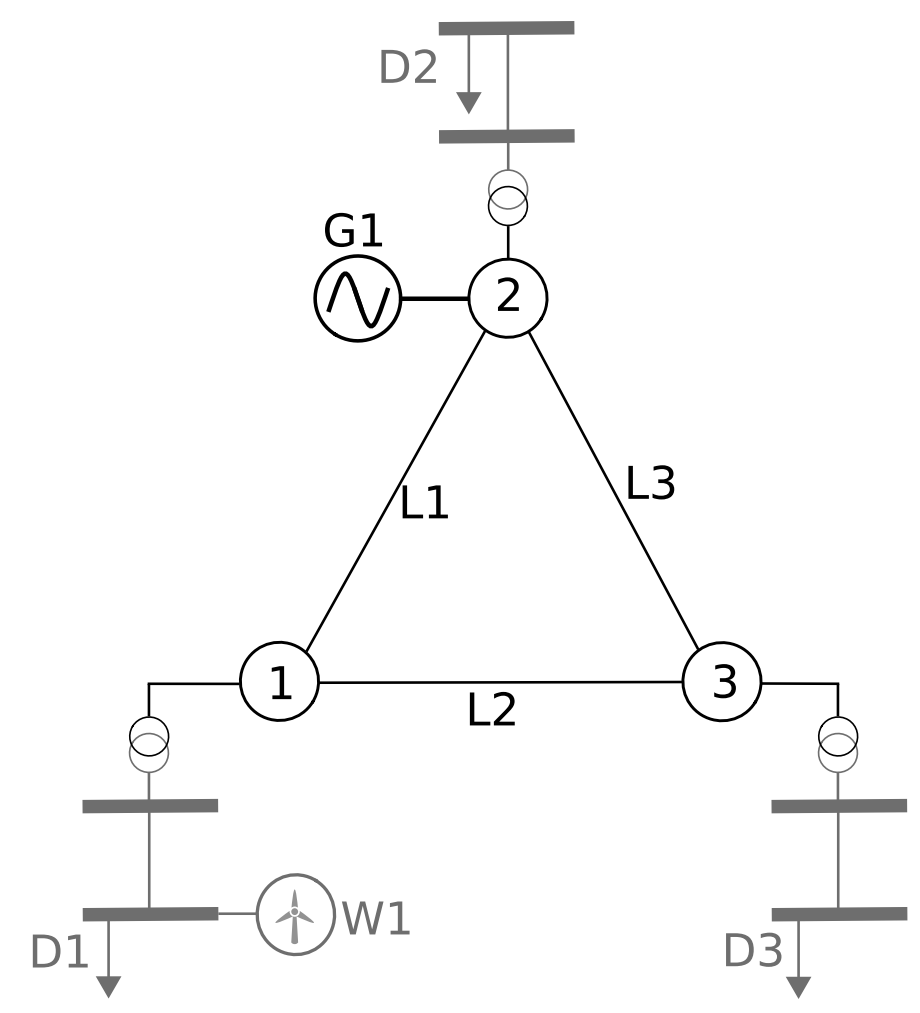

Figure 3: Topology of the small-scale illustrative case study.

For an upper bound (ideal benchmark), we consider a full coordination between TSO and DSOs such that both have full information of real-time scenarios in the day-ahead stage. This ideal benchmark is a co-optimization problem formulated as an SOCP:

$$
\begin{array}{rl}
\max _{\widehat{p}_{g}^{\mathrm{DA}}, \widehat{p}_{d}^{\mathrm{DA}}, s^{\mathrm{DA}}, w_{r}^{\mathrm{DA}}, \Xi^{\mathrm{RT}}} & \mathcal{S} \mathcal{W}^{\mathrm{DA}}-\sum_{\omega} \phi_{\omega} \Delta \mathrm{Cost}_{\omega}^{\mathrm{RT}} \\
\text { subject to: } & (3 \mathrm{~b})-(3 \mathrm{~h}) \text { and }(4 \mathrm{~b})-(4 \mathrm{w}) .
\end{array}
$$

This full coordination is however quite impractical as it requires TSO and DSOs to solve a common single problem, and all information is packed into one optimization problem, which quickly becomes intractable for larger systems, while being incompatible with the separation of DSO and TSO roles in the European market.

To obtain a lower bound for the expected social welfare, we consider a model with no coordination, and solve day-ahead and real-time models sequentially. This means that we first solve the day-ahead market (3) without imposing any cap on the quantity bids of DSO-level DERs. Then, we solve the real-time problem (4) for each scenario separately, i.e., in a deterministic manner. We then calculate the resultant total expected social welfare. 


\begin{tabular}{c|ccc} 
& Resistance [p.u.] & Reactance [p.u.] & Capacity [MVA] \\
\hline TSO-level line L1 & n/a & 0.01 & 35 \\
TSO-level line L2 & n/a & 0.03 & 60 \\
TSO-level line L3 & n/a & 0.01 & 60 \\
Interface at node 1 & 0 & 0 & 200 \\
Interface at node 2 & 0 & 0 & 200 \\
Interface at node 3 & 0 & 0 & 200 \\
Every line within distribution systems & 0.002 & 0.001 & 200
\end{tabular}

Table 1: Network data in the small-scale illustrative case study. The resistance values for transmission lines are not necessary, since a lossless linear power flow approximation is used in the transmission level.

\begin{tabular}{c|ccccc} 
& $\bar{P}_{g / d}[\mathrm{MW}]$ & $\bar{Q}_{g / d}[\mathrm{MVAr}]$ & $\pi_{g / d}^{\mathrm{DA}}[\$ / \mathrm{MWh}]$ & $\pi_{g / d}^{\uparrow}[\$ / \mathrm{MWh}]$ & $\pi_{g / d}^{\downarrow}[\$ / \mathrm{MWh}]$ \\
\hline G1 & 1000 & 200 & 5 & 5 & 6 \\
D1 & 165 & 50 & 19 & 8 & 8 \\
D2 & 165 & 50 & 19 & 8 & 8 \\
D3 & 165 & 50 & 19 & 8 & 8 \\
W1 & 150 & 0 & 0 & 10 & 10
\end{tabular}

Table 2: Technical data for generator G1, loads D1, D2, and D3, as well as wind power unit W1 in the small-scale illustrative case study. The lower bound for production/consumption level of all agents is assumed to be zero. The installed capacity of wind power unit W1 is $150 \mathrm{MW}$. This unit bids to the day-ahead market a mean forecast, which is $115.4 \mathrm{MW}$.

\section{Case study}

The proposed bi-level problem (2) with a solution strategy relying on multi-cut Benders' decomposition as explained in the previous section is implemented in Matlab using CVX and solved with MOSEK 8.0. The Benders' decomposition is set to converge if the upper and lower bounds come to within a relative gap of $0.1 \%$. A speed-up is achieved by solving the sub-problems, one per scenario, in parallel ${ }^{15}$. In the rest of this section, we first provide a small-scale illustrative example, and then present the coordination results for a larger case study.

\subsection{Illustrative case study}

In this section we showcase the concept of the interface optimizer using a small-scale illustrative case study with a three-node transmission network, connected to three DSOs. The topology of this system is shown in Fig. 3. A TSO operates the transmission system, including nodes 1, 2, and 3, as well as transmission lines L1, L2, and L3. The network data is available in Table 1. Transmission line L1 has the lowest capacity (35 MVA). Note that this is a made-up illustrative example, and does not necessarily represent a real-world power system. A realistic case study will be presented subsequently in Section 5.2

A flexible conventional generator, namely G1, is located in the operational domain of the TSO, connected to node 2. Each of the three nodes in the transmission network is connected to a twonode distribution system, operated by its own DSO. Demands D1, D2, and D3 are located in

\footnotetext{
${ }^{15}$ Hardware used: Huawei XH620 V3, 2x Intel Xeon Processor 2650v4 (12 core, 2.20GHz), 256 GB memory, 480 GB-SSD disk.
} 


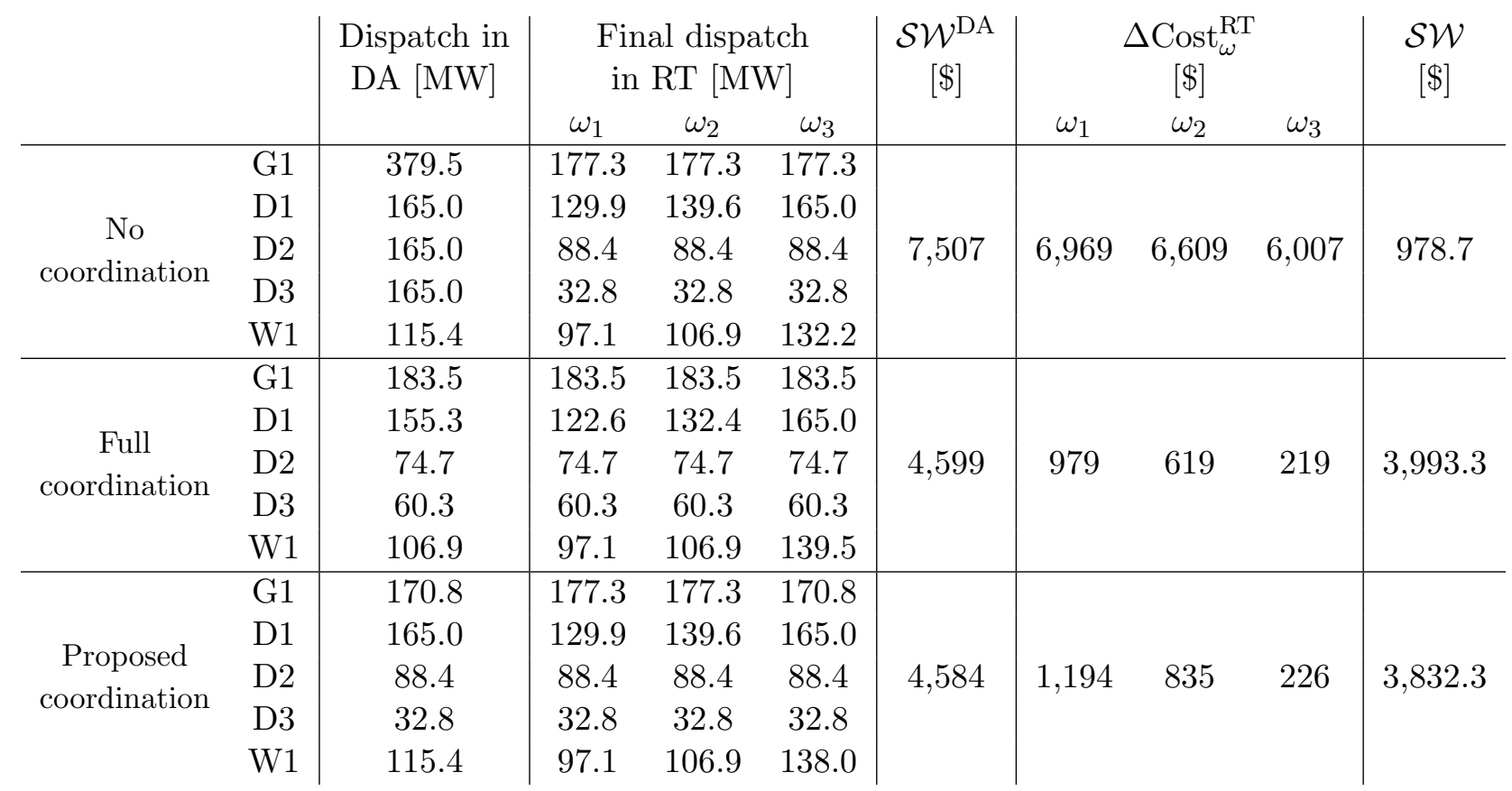

Table 3: Results for the small-scale illustrative case study. DA: day-ahead; RT: real-time.

distribution systems, each in the operational domain of a different DSO. In addition, there is a wind power unit, namely $\mathrm{W} 1$, connected to one of distribution systems. The generation of this wind power unit is the sole source of uncertainty, modeled by three equiprobable scenarios $\omega_{1}, \omega_{2}$, and $\omega_{3}$. According to the wind power forecast in the day-ahead stage, three scenarios for power production of this unit are 97.1 MW, 106.9 MW, and 142.2 MW. The quantity bid production of this unit in the day-ahead market is the mean of production forecast, i.e., 115.4 MW. Accordingly, there is a wind power deficit of $18.3 \mathrm{MW}$ under scenario $\omega_{1}$, a wind power deficit of $8.5 \mathrm{MW}$ under scenario $\omega_{2}$, and a wind power excess of $26.8 \mathrm{MW}$ under scenario $\omega_{3}$.

The quantity bids as well as the bid prices of all generators and demands are given in Table 2 . The day-ahead bid price of the wind power unit W1 is zero, although it has the highest bid price among all agents for the regulation service provision in the real-time stage. The value of lost load in the case of involuntarily load curtailment is $\$ 10,000 / \mathrm{MWh}$.

Table 3 provides the market-clearing outcomes for three TSO-DSO coordination mechanisms, i.e., a case with no coordination, the full coordination obtained by the TSO-DSO co-optimization (ideal benchmark), and the proposed coordination through the interface optimizer. Four columns of Table 3 show the day-ahead dispatch of agents (i.e., $\widehat{p}_{g}^{\mathrm{DA}}, \widehat{p}_{d}^{\mathrm{DA}}$, and $w_{r}^{\mathrm{DA}}$ ), their final dispatch in the real-time stage under three scenarios (i.e., $p_{g \omega}^{\mathrm{RT}}, p_{d \omega}^{\mathrm{RT}}$, and $w_{r \omega}^{\mathrm{RT}}$ ), the social welfare in the day-ahead stage, and the social cost in the real-time stage under each scenario, respectively.

Let us start analyzing the market results for the case with no coordination. In this case, the day-ahead market is fully unaware of wind power scenarios and network constraints. Due to this naive decision-making process, all demands D1, D2, and D3 are dispatched at their maximum consumption level, i.e., $165 \mathrm{MW}$. In addition, the wind power unit who bids at zero price is fully dispatched at its day-ahead quantity bid, i.e., 115.4 MW. This results in the maximum day-ahead social welfare among three coordination mechanisms, that is $\$ 7,507$. However, these day-ahead 
market-clearing outcomes are infeasible in the operational stage when the network constrained are enforced. The TSO requires procuring a significant amount of regulation services to re-dispatch the day-ahead schedules to make the market outcomes feasible and to offset the wind power deviations in the real-time stage, causing a remarkable social cost. The total expected social welfare, i.e., $\mathcal{S} \mathcal{W}^{\mathrm{DA}}-\frac{1}{3}\left[\Delta \operatorname{Cost}_{\omega_{1}}^{\mathrm{RT}}+\Delta \operatorname{Cost}_{\omega_{2}}^{\mathrm{RT}}+\Delta \operatorname{Cost}_{\omega_{3}}^{\mathrm{RT}}\right]$, in this case is $\$ 978.7$, which is the lowest value among three mechanisms.

The full coordination mechanism provides a perfect coordination between the day-ahead and real-time markets (temporal coordination) and between the TSO and DSOs (sectoral coordination). In this mechanism, the day-ahead market clearing is fully aware of network constraints and wind power scenarios. Therefore, all agents are dispatched in the day-ahead stage, such that the TSO and DSOs procure small amount of regulation services in the real-time stage to offset wind power deviations while operating their underlying system safely. In this mechanism, the total expected social welfare is $\$ 3,993.3$, that is the highest value among three coordination mechanisms.

The proposed coordination mechanism via interface optimizer is able to successfully approximate the full coordination mechanism, although the day-ahead market in this mechanism is unaware of both network constraints and wind power scenarios. The total expected social welfare in this mechanism is $\$ 3,832.3$, which is only $4 \%$ lower than that in the ideal benchmark. In this mechanism, two out of three DSOs impose caps on the day-ahead quantity bid of demands D2 and D3, such that these two demands are partially supplied in the day-ahead stage. In detail, their day-ahead dispatch are 88.4 MW and 32.8 MW, while they intended to bid $165 \mathrm{MW}$ each without such caps. In this specific case, similar to demand D1, the day-ahead quantity bid of the wind power unit is not capped.

The bottom line is that the proposed coordination mechanism respects the current European market regulations by taking into account a deterministic setup for the day-ahead market clearing, where the transmission constraints are excluded or minimally represented. Nevertheless, it achieves the market-clearing outcomes close to those of the ideal benchmark by imposing caps on the quantity bids of the DSO-level flexible resources to the day-ahead market.

\subsection{Case study with IEEE 24-node test system}

\subsubsection{Input data}

A modified version of the IEEE 24-node reliability test system (Ordoudis et al., 2016) is used. The network is extended with the addition of five radial distribution feeders, which replace the loads at nodes $6,13,15,18$, and 19 . This implies that a single TSO interacts with five DSOs (namely, DSO 1 to DSO 5). In total, $39.3 \%$ of the total system load is placed in radial distribution systems operated by DSOs, while the remaining of the total load is connected to the transmission grid. A diagram of the entire network is given in Appendix B. The system is also equipped with seven additional wind farms, which are the sole sources of uncertainty in the system. One of the wind power units is located in a distribution system operated by DSO 3, while the rest of wind power units are connected to the transmission grid. In order to have some congestion in the network, the capacity of several TSO-level lines and generator capacities are changed. All the network and generator/demand data necessary to replicate the case study is available in the online appendix (Hermann et al., 2021).

The scenarios for seven wind farms are generated by random sampling from a Gaussian mixture model, whose covariance is based on the geographical distance of the wind farms to each other. For a more in-depth description of the scenario generation method, we refer to Appendix A. It 
is outside the scope of this paper to use more advanced forecasting tools to generate scenarios without taking an assumption on the known probability distribution of the uncertainty ${ }^{16}$.

From the scenarios generated, we first arbitrarily pick 20 in-sample scenarios to solve the proposed bi-level model, and then select different 300 scenarios for an ex-post out-of-sample validation. All scenarios are given in the online appendix (Hermann et al., 2021). We assign the same probability to all scenarios. For the in-sample analysis, the interface optimizer solves the proposed bi-level problem using the arbitrarily selected 20 in-sample scenarios to determine the optimal values of coordination variables at all five interfaces. For fixed day-ahead decisions obtained from the in-sample analysis, the real-time market is cleared deterministically for each of 300 out-of-sample scenarios (the so-called out-of-sample simulations). We calculate the expected social welfare by deducting the mean real-time social cost (obtained from the out-of-sample simulations) from the day-ahead social welfare (obtained from the in-sample analysis). This value under different market setups will be reported in the next section.

\subsubsection{Results for the whole system: coordination under increasing wind power penetration}

In order to examine the improvement of the social welfare achieved by the proposed TSO-DSO coordination mechanism, we simulate the system with increasing wind power penetration ${ }^{17}$. We do this by uniformly scaling the nameplate capacity of each of the seven wind farms, thereby increasing the wind power penetration. In particular, we simulate for 20 different wind power penetration levels, ranging from $10 \%$ to $150 \%$.

As the results of the out-of-sample simulations, Fig. 4 presents the expected social welfare obtained from the proposed coordination method via interface (PCC) optimizer along with two benchmark models. The 0.8 quantile and the 0.2 quantile are also given in this figure to show the spread of each curve resulting from the uncertainties. It also includes a heat-map indicating the level of transmission network congestion. We define "congestion level" as the probability of at least two transmission lines being congested in the real-time stage. There is a strong connection between the expected social welfare and the wind power penetration. For all three models, the slope of the expected social welfare changes in the range between $40 \%$ and $80 \%$ wind penetration, where the congestion in the transmission network also starts to affect the system. Comparing the expected social welfare obtained in the proposed coordination model versus two benchmarks, the interface optimizer improves the expected social welfare compared to the benchmark with no coordination. Clearly, the interface optimizer does not necessarily perform as efficient as the ideal benchmark, because the ideal benchmark can take advantage of the TSO-level resources in the day-ahead market to meet grid constraints, whereas the interface optimizer relies on the DSO-level resources only. In the deterministic dispatch model (uncoordinated benchmark), the expected social welfare diminishes after $75 \%$ wind penetration due to the system running out of available flexible resources. This happens because the day-ahead stage clears the market mostly with wind power as it is cheap, however the balancing measures in the real-time stage are costly, reducing the overall social welfare. The ideal stochastic dispatch can mitigate this situation by reducing the cleared wind power in the day-ahead stage and the expected social welfare will not reduce with

\footnotetext{
${ }^{16}$ We refer the interested reader to Pinson et al. (2009) for more advanced scenario generation tools. We also refer to Maggioni et al. (2019) for exploring the value of choosing right distribution in stochastic programming.

${ }^{17}$ We define the wind power penetration as the total forecasted available wind power in the day-ahead stage divided by the total quantity bids of demands, i.e., $\frac{\sum_{r} W_{r}^{\mathrm{DA}}}{\sum_{d} \bar{P}_{d}} \times 100$. Clearly, this value could exceed $100 \%$, but at most $100 \%$ of demands in the day-ahead stage can be supplied by wind farms.
} 


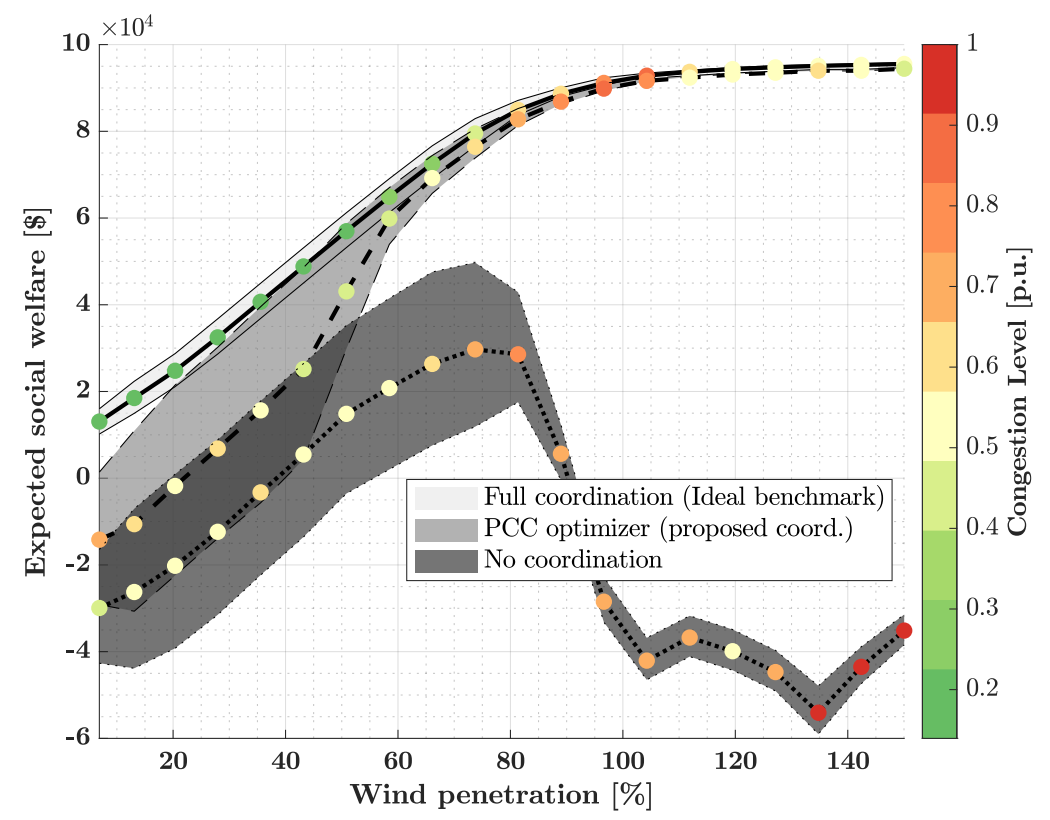

Figure 4: The expected social welfare as a function of wind power penetration obtained from the proposed coordination model, i.e., interface (PCC) optimizer, and two benchmark models. The 0.2 quantile and 0.8 quantile are also depicted. The colored dots indicate the level of congestion in the transmission network as the probability of at least two lines being congested in the real-time stage. The results are obtained from the out-of-sample simulations.

increasing wind penetration. As evidenced by Fig. 4, the interface optimizer manages to partially reduce the need for expensive balancing actions.

The results of Fig. 4 clearly show that the proposed coordination model could successfully approximate the ideal benchmark by allowing DSOs to impose caps on the bids of distributed energy resources. Following the current practice in the European day-ahead electricity market, recall that the day-ahead market-clearing problem in the proposed coordination model is deterministic and copper-plate (meaning that all network constraints are excluded). One may hypothesize that the caps on the bids of the distributed energy resources implicitly make the proposed coordination model aware of network constraints and uncertainties. To explore this hypothesis, we develop two additional benchmarks, built upon the uncoordinated model. The first one, the so-called networkaware benchmark, refers to the sequential day-ahead and real-time markets, where the network constraints in both TSO and DSO levels are fully included in the day-ahead stage. Similarly, the second model, the so-called uncertainty-aware benchmark, is a sequential day-ahead and real-time market-clearing setting, in which the day-ahead market solves a stochastic model including all 20 in-sample scenarios. The curves of expected social welfare as a function of wind power penetration obtained from five models are given in Fig. 5, including those three already shown in Fig. 4 as well as the results of network- and uncertainty-aware benchmarks. These results show that the network constraints play a key role, such that an uncoordinated but network-aware model provides similar results to those of ideal benchmark and the proposed model. The uncertainty awareness can also enhance the performance of the uncoordinated model, but the improvement is not comparable to the case with the network awareness. The results of Fig. 5 stresses the success of the proposed coordination model to bring network- and uncertainty-related information to the day-ahead market 


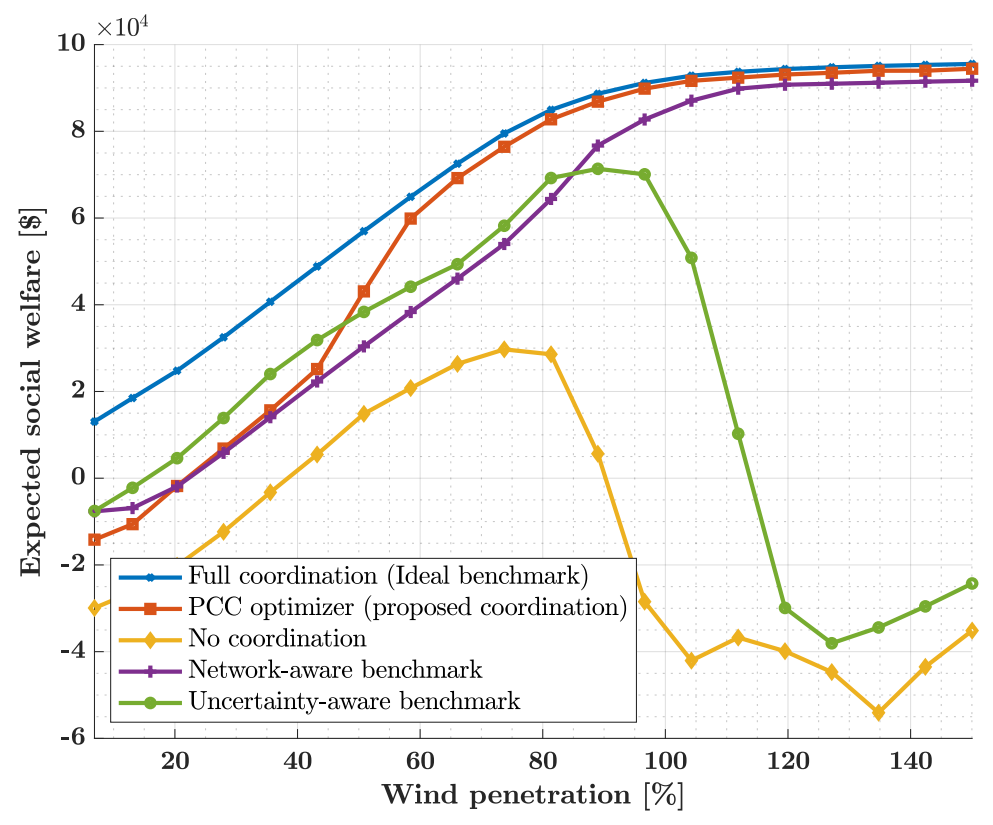

Figure 5: The expected social welfare as a function of wind power penetration obtained from five models, including those three in Fig. 4 as well as two additional models, namely network-aware and uncertainty-aware benchmarks. The results are obtained from the out-of-sample simulations.

in an implicit way through imposing optimal caps, while respecting the current regulations.

\subsubsection{Measuring the effect of increasing the number of in-sample scenarios}

To explore the impacts of in-sample scenarios fed to our proposed coordination model, we vary the number of those scenarios from 1 to 40, all arbitrarily picked. The 300 out-of-sample scenarios are unchanged. The upper plot in Fig. 6 illustrates the expected in-sample and out-of-sample social welfare as a function of the number of in-sample scenarios. The 0.9 quantile and the 0.1 quantile of the out-of-sample social welfare are also given in this figure. As expected, the social welfare is very sensitive to in-sample scenarios when the number of those scenarios is comparatively low. However, both in-sample and out-of-sample curves as well as quantiles stabilize when the number of in-sample scenarios reaches 15 . In addition, the difference between the expected in-sample and the expected out-of-sample social welfare values is negligible. This confirms our choice in selecting 20 in-sample scenarios for this case study.

To further analyze the importance of uncertainty modeling in the proposed coordination mechanism, we use two well-known measures in stochastic programming, namely the Value of Stochastic Solution (VSS) and the Expected Value of Perfect Information (EVPI) (Conejo et al., 2010). For the VSS, we solve the proposed coordination model two times, once deterministically with a single scenario representing the mean of in-sample scenarios, and once stochastically with the given set of in-sample scenarios. The absolute difference between the expected in-sample social welfare obtained from the stochastic problem and the in-sample social welfare obtained from the deterministic problem gives the VSS. Unlike the VSS that we solve two problems (one deterministic and one stochastic), we solve $K+1$ problems to compute the EVPI, where $K$ is the number of in-sample scenarios. For each of the $K$ in-sample scenarios, we solve the proposed coordination model deter- 


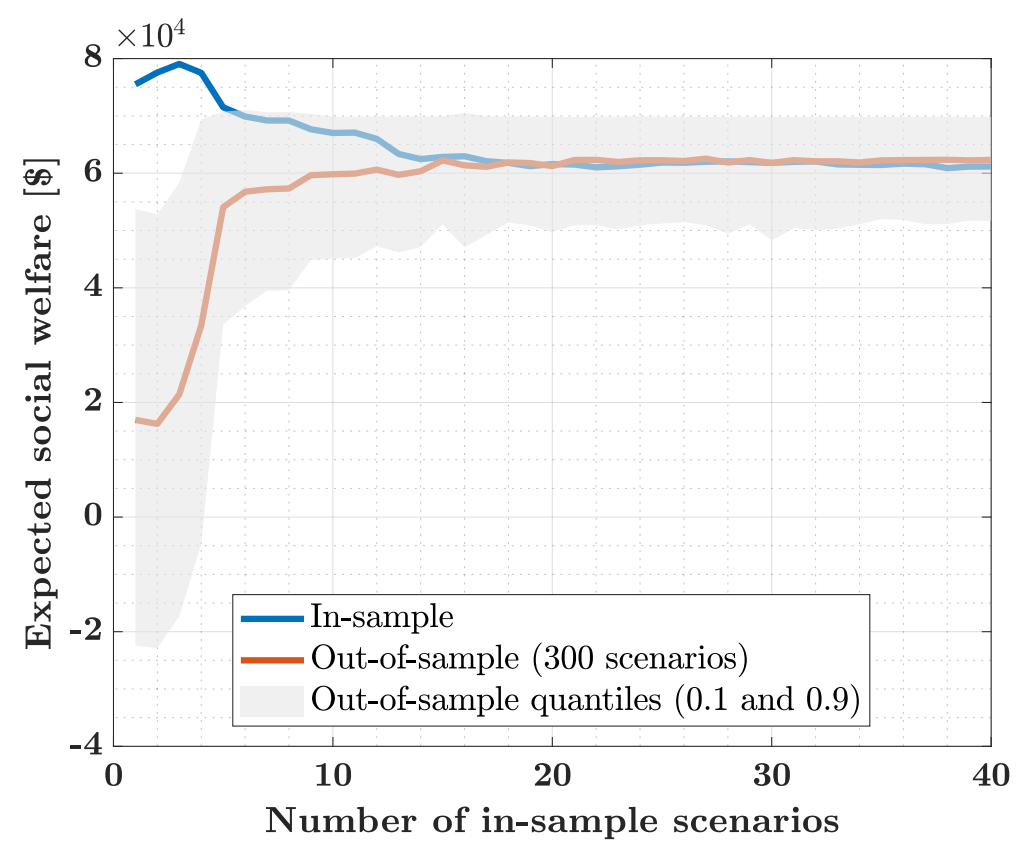

(a) The expected social welfare as a function of the number of in-sample scenarios.

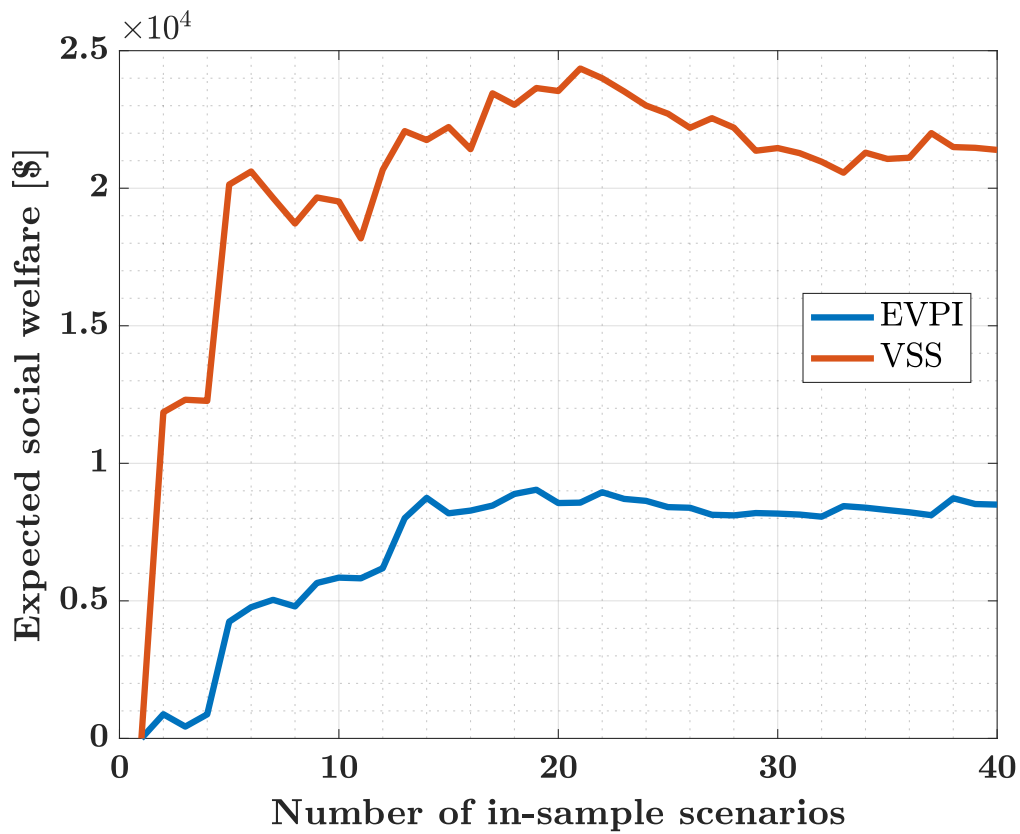

(b) The VSS and EVPI as a function of the number of in-sample scenarios.

Figure 6: Measuring the effect of increasing the number of in-sample scenarios. The wind power penetration is fixed to $60 \%$.

ministically. We also solve the problem stochastically with the given $K$ number of scenarios. The absolute difference between the average in-sample social welfare obtained from $K$ deterministic problems and the expected in-sample social welfare achieved from the stochastic model with $K$ 
in-sample scenarios gives the EVPI. The lower plot in Fig. 6 shows the values of VSS and EVPI as a function of the number of in-sample scenarios. The values achieved for both VSS and EVPI over different number of in-sample scenarios are remarkable, showing the importance of modeling uncertainty. For example, let us focus on a case with 20 in-sample scenarios. The expected out-ofsample social welfare (the upper plot in Fig. 6) is nearly $\$ 60,000$, whereas the value obtained for the VSS (the lower plot) is approximately $\$ 24,000$, i.e., $40 \%$ of the expected social welfare. For this case, the EVPI is $\$ 8,500$, which is $14 \%$ of the expected social welfare. All these numbers highlight the important role of the uncertainty.

\subsubsection{Results for the interfaces: physical capacity versus optimal limit}

This section provides some insight into the power flow at five interfaces resulting from the proposed coordination model and two benchmarks, i.e., full coordination and no coordination. For various levels of wind power penetration, Fig. 7 depicts the apparent power flow in both dayahead and real-time trading stages in one of the interfaces, i.e., the one between TSO and DSO 2. The three blue bars (dark to light) provide the deterministic power flow in the day-ahead stage, achieved from the proposed coordination method, and that in two benchmarks. Each black bar in the center of each blue bar indicates the expected real-time power flow at the interface across 300 out-of-sample scenarios. Under each out-of-sample scenario, the power flow at the interface is constrained by the physical limit of the interface. In addition to the expected value of the real-time power flow, the 0.1 and 0.9 quantile are also given.

An interesting observation is that the interface optimizer imposes a cap on the apparent power flow at the interface of TSO and DSO 2 in the day-ahead stage, which is tighter than the physical limit (500 MVA). By this cap, the interface optimizer restricts the participation of distributed energy resources located at the operational domain of DSO 2 in the wholesale TSO-level market. As discussed in Section 5.2.2., one can interpret this cap as an effective means to make the deterministic copper-plate day-ahead market aware of network constraints and uncertainty.

The relation of the optimal caps to the network model is highly non-linear, as demonstrated in Fig. 8. Here, the physical capacity of the interface between TSO and DSO 1 is varied, and the resulting optimal caps are found. This figure shows that the interface optimizer is highly reliant on having access to precise network information, and that the decisions of one DSO can affect even other DSOs' optimal dispatch of flexible resources.

\subsubsection{Computational performance}

For this case study, the bi-level model (2) is solved using the multi-cut Benders' decomposition on average within 14 minutes. More details are provided in Table 4. The results of this table are the average values obtained from 20 different in-sample simulations, each with a different level of wind power penetration. Since the sub-problems, one per in-sample scenario, are independent and can be solved in parallel, the number of scenarios do not affect the computational time, as long as there are enough CPU cores to solve them in parallel. Fig. 9 presents the convergence of the proposed multicut Benders' decomposition for a sample level of wind power penetration. A relevant observation is that the convergence of upper and lower bounds seems to be rather smooth, except a few spikes in the upper bound. If significant fluctuations occur, we recommend using a regularized Benders' decomposition (Ruszczynski \& Swietanowski, 1997). 


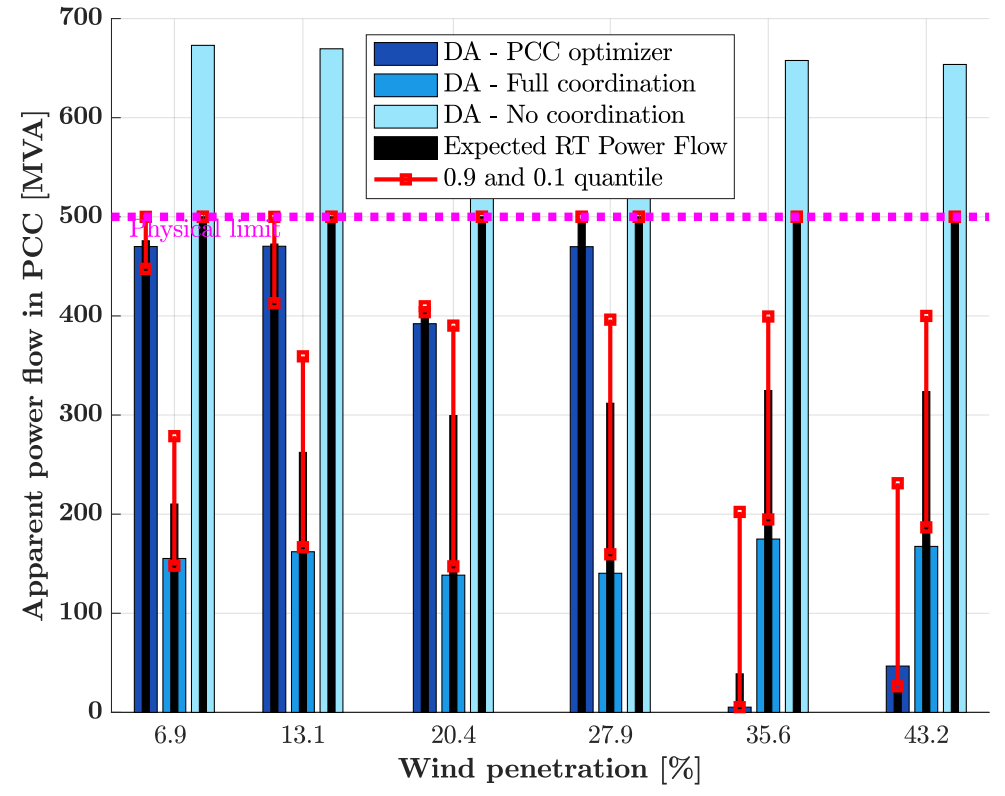

Figure 7: The apparent power flow at the interface of TSO and DSO 2 (connected to node 6) in both day-ahead and real-time trading stages. The real-time results are the outcomes of the out-of-sample simulation. These results are obtained from the proposed coordination method through interface (PCC) optimizer, and two benchmark models, i.e., full coordination and no coordination.

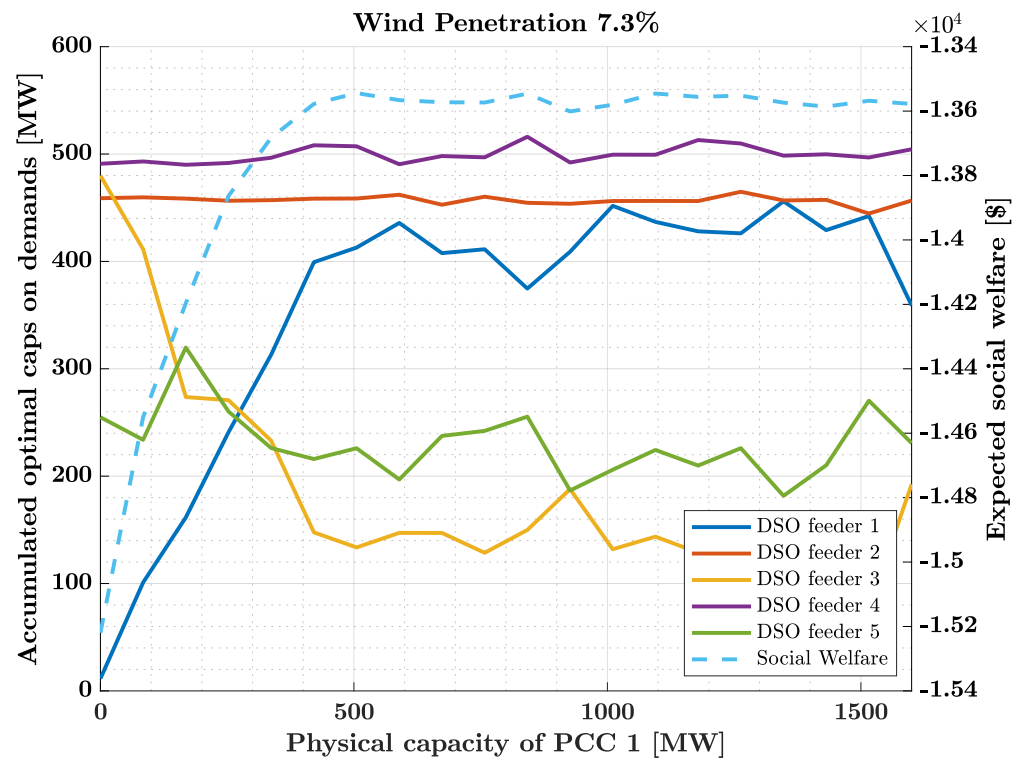

Figure 8: The optimal caps of the interface optimizer on the quantity bid of aggregate flexible loads in different feeders as a function of the physical capacity of interface between TSO and DSO 1 (note: the wind penetration is fixed to $7.3 \%)$. 


\begin{tabular}{ccccc}
\hline $\begin{array}{c}\text { Average time } \\
\text { to solve } \\
\text { each subproblem }\end{array}$ & $\begin{array}{c}\text { Average time } \\
\text { to solve } \\
\text { master problem }\end{array}$ & $\begin{array}{c}\text { Master problem } \\
\text { in the first } \\
\text { iteration }\end{array}$ & $\begin{array}{c}\text { Master problem } \\
\text { in the last } \\
\text { iteration }\end{array}$ & $\begin{array}{c}\text { Average } \\
\text { number of } \\
\text { iterations }\end{array}$ \\
\hline $0.40 \mathrm{~s}$ & $0.75 \mathrm{~s}$ & $0.72 \mathrm{~s}$ & $1.00 \mathrm{~s}$ & 27.3 \\
\hline
\end{tabular}

Table 4: Computational performance of the proposed multi-cut Benders' algorithm. Columns 1-4 demonstrate the CPU time in seconds. The last column shows the number of iterations. This table provides the average results obtained from 20 different in-sample simulations, each with a different level of wind power penetration.

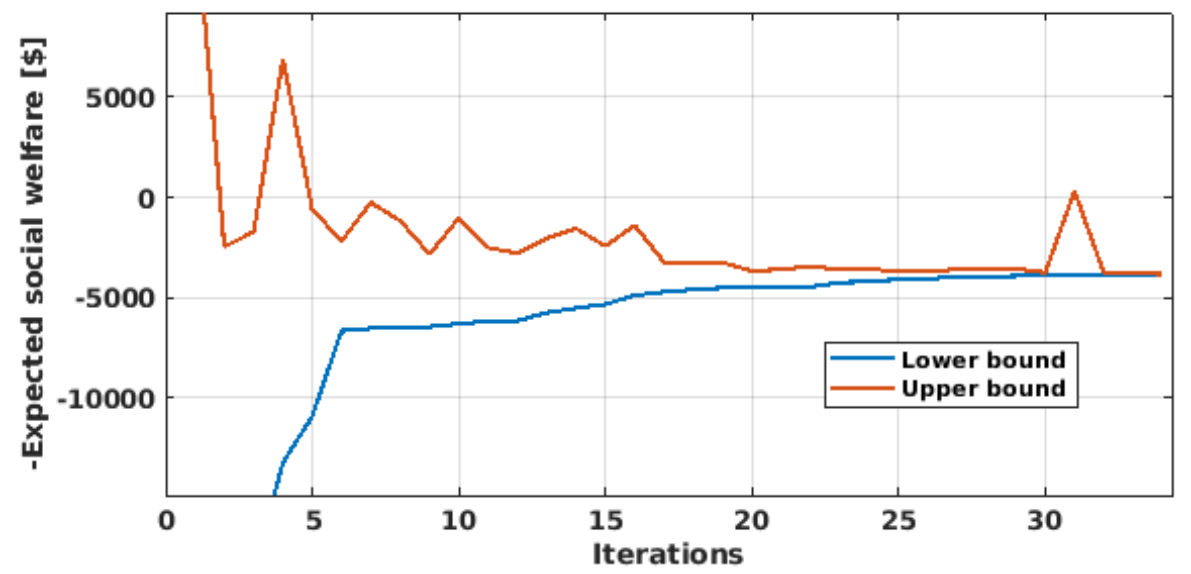

Figure 9: Convergence of the multicut Benders' decomposition implemented to the proposed stochastic bi-level problem (2). The vertical axis shows the expected social cost (or minus expected social welfare) in the real-time stage.

\section{Conclusion}

The optimal day-ahead schedule for DSO-level flexible resources was modeled through a Stackelberg game, cast as a bi-level problem. The interface optimizer acting as a leader in the game can provide prices and capacity limits at the interface which lead localized DSO flexibility markets to approximate the stochastic ideal dispatch. Our findings have implications for designing practical mechanisms to use this avenue of TSO-DSO coordination, in two ways: First, the optimal interface capacity for coordination is congruent with, but not limited by, the physical capacity of the underlying hardware. Thus, any mechanism should take as guideline the physical interface capacity but must not be limited by it. Second, the optimal caps for one feeder can depend in a non-obvious way on the physical capacity of another feeder or the physical capacity of the transmission network. Therefore, when considering to implement a mechanism to coordinate day-ahead dispatches in the manner analyzed here, the sensitivity of the outcomes with respect to the underlying network data should be analyzed.

We find out that under an assumption of information symmetry between interface optimizer and DSO, a mathematical equivalence exists between local DSO flexibility markets and global (stochastic) markets. This makes for an interesting observation: The implementation of localized DSO markets can lead to an approximation of the stochastic ideal dispatch, given an appropriate coordination mechanism. 
This work uses optimal real-time coordination to highlight differences in the day-ahead dispatch coordination. However, it is an unrealistic position, due to separation of responsibilities and knowledge between DSO and TSO, as evidenced by the previous works examining methods for real-time coordination. This highlights the need for some afterthought when implementing policies on coordination: Which time stage or which combination of day-ahead and real-time coordination is appropriate?

As a general point, the inclusion of day-ahead coordination is complementary to real-time coordination. Any real-time coordination method that increases the system social welfare may overestimate improvements if compared under uncoordinated day-ahead dispatches. Further, coordination in the day-ahead stage will generally increase the social welfare as premiums are applied closer to real-time. Improving coordination in the day-ahead stage will reduce the needed amount of real-time re-dispatching, benefitting the end-users.

As potential future works, it is of interest to relax the assumptions made in this work. Additional sources of uncertainty need to be considered, although this will increase the number of scenarios and therefore the computational burden. One can think of alternative ways to model uncertainty. For example, one potential alternative is to use chance-constrained programming, resulting in a chance-constrained electricity market design (Dvorkin, 2020). In addition, it is relevant to relax the assumption of information symmetry among the market players (Dvorkin et al., 2019b), which are the interface optimizer, TSO and DSOs in this study, and explore how the resulting model can be solved and how asymmetric information among players impacts the overall social welfare. Aiming to develop a more realistic model, it is important to include inter-temporal operational constraints and additional market products in future works.

\section{Appendix A. Scenario generation}

A simple scenario generation method is used for characterizing the wind power uncertainty. Let $r=\{1, \ldots,|R|\}$ and $s=\{1, \ldots,|R|\}$ denote the set of wind farms, where $|R|$ is the number of wind farms. This number in our case study is 7 . The geographical location of each wind farm $r$ is defined by its X-coordinate $X_{r}$ and Y-coordinate $Y_{r}$. The geographical distance of each two wind farms $r$ and $s$ is

$$
D_{r s}=\left\|\begin{array}{c}
X_{r}-X_{s} \\
Y_{r}-Y_{s}
\end{array}\right\|_{2}, \quad \forall r \in R, s \in R .
$$

Let $\sigma_{r}^{2}$ denote the variance of wind power generation of farm $r$, captured from the historical data. The co-variance matrix $\boldsymbol{\Sigma}$ can now be constructed, whose element $\Sigma_{r s}$ indicating the spatial correlation between farms $r$ and $s$ is

$$
\Sigma_{r s}=\sqrt{\sigma_{r}^{2} \sigma_{s}^{2}}\left(\frac{1+\kappa}{e^{D_{r s}}}-\kappa\right), \forall r \in R, s \in R,
$$

where $e$ is the Euler number, and $\kappa$ is a dimensionless distance correlation factor of appropriate size to be chosen. Defining the co-variance matrix as in (A.2) will ensure that it is positive semidefinite, i.e., all eigenvalues are non-negative. The wind power distribution of each farm is assumed to be Gaussian, i.e., $W \sim \mathcal{N}(\mu, \boldsymbol{\Sigma})$, where $\mu$ is the mean power production vector, captured from the historical data. Given this Gaussian distribution, all scenarios $\omega \in \Omega$ are generated by random sampling. 


\section{Appendix B. Additional details on the modified IEEE 24-node test system}

Fig. B.10 depicts the topology of the modified IEEE 24-node reliability test system (Ordoudis et al., 2016), including five distributions systems, operated by DSO 1 to DSO 5. The mean and variance of wind power forecast for all seven wind power units are given in Table B.5. The geographical location of each wind power unit is given in Table B.6, which is used to calculate the distance between them as previously used in (A.1) for scenario generation.

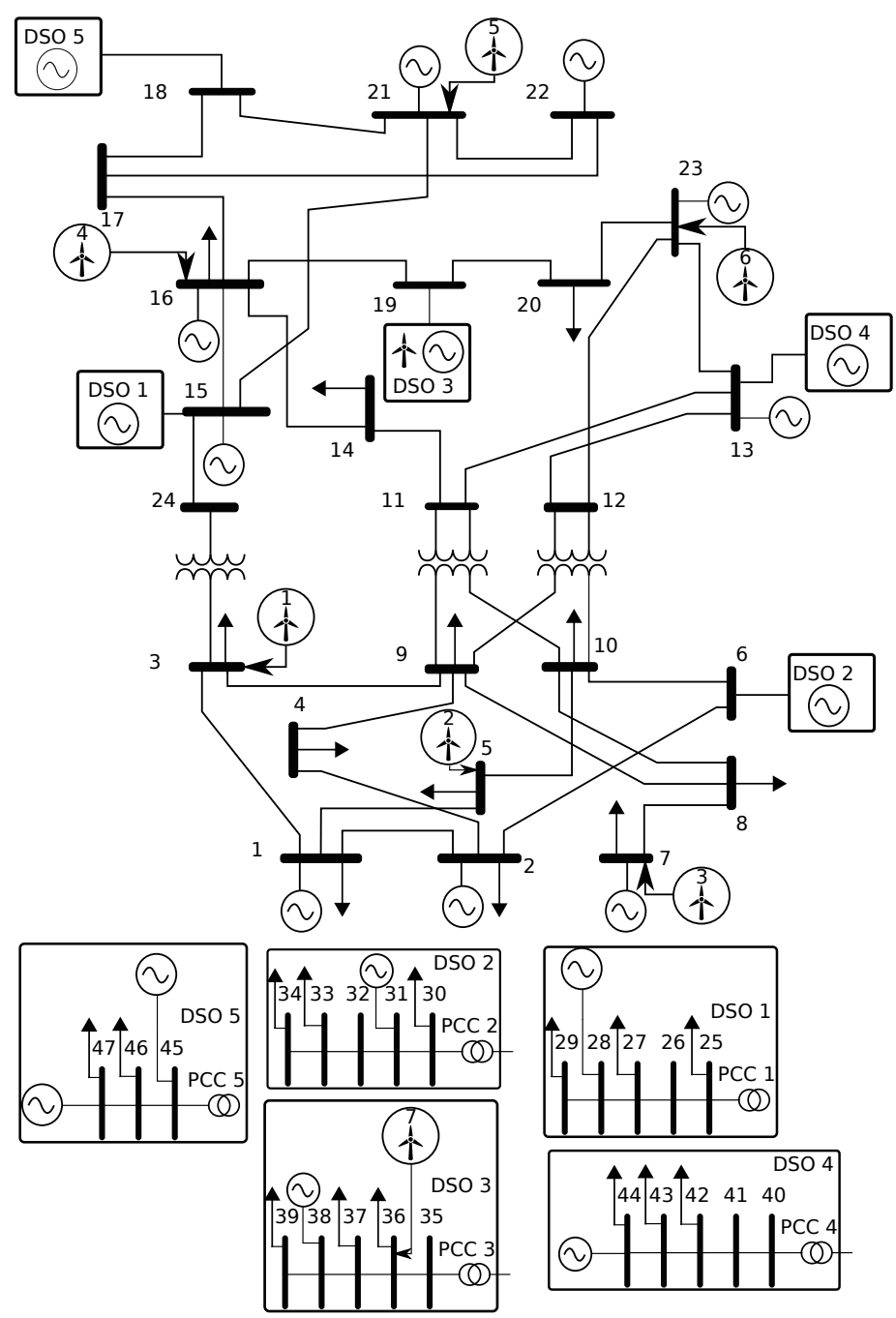

Figure B.10: Diagram of the modified IEEE 24-node reliability test system (Ordoudis et al., 2016), including five distributions systems connected to the main transmission grid.

Table B.5: The mean and standard deviation of wind power generation for seven farms.

\begin{tabular}{c|ccccccc} 
& 1 & 2 & 3 & 4 & 5 & 6 & 7 \\
\hline Mean $\mu$ & 1429 & 1429 & 857 & 571 & 571 & 285 & 571 \\
Standard deviation $\sqrt{\sigma^{2}}$ & 142 & 142 & 86 & 57 & 57 & 29 & 57
\end{tabular}


Table B.6: The geographical location of seven wind farms.

\begin{tabular}{c|ccccccc} 
& 1 & 2 & 3 & 4 & 5 & 6 & 7 \\
\hline X-coordinate & 0 & 0.25 & 1.5 & 1 & 1.25 & 1.5 & 1.75 \\
Y-coordinate & 0 & 0 & 0 & 3 & 3 & 4 & 4.2
\end{tabular}

\section{References}

Aravena, I., \& Papavasiliou, A. (2017). Renewable energy integration in zonal markets. IEEE Transactions on Power Systems, 32, 1334-1349.

Bai, L., Wang, J., Wang, C., Chen, C., \& Li, F. (2018). Distribution locational marginal pricing (DLMP) for congestion management and voltage support. IEEE Transactions on Power Systems, 33, 4061-4073.

Benders, J. F. (1962). Partitioning procedures for solving mixed-variables programming problems. Numerische Mathematik, 4, 238-252.

Van den Bergh, K., Boury, J., \& Delarue, E. (2016). The flow-based market coupling in central western Europe: Concepts and definitions. The Electricity Journal, 29, 24-29.

Birge, J., \& Louveaux, F. (1988). A multicut algorithm for two-stage stochastic linear programs. European Journal of Operational Research, 34, 348-392.

Bobo, L., Venzke, A., \& Chatzivasileiadis, S. (2021). Second-order cone relaxations of the optimal power flow for active distribution grids: Comparison of methods. International Journal of Electrical Power 83 Energy Systems, 127. Article number: 106625.

Brath, P., Gehrke, O., \& Morales Bondy, D. (2019). DSO's Role in Electricity Market (DREM) Project. Technical Report. URL: https://www.ienergi.dk/sites/ienergi.dk/files/media/dokumenter/2019-04/ ResultaterfraDREM-projektet.pdf.

Callaway, D. S., \& Hiskens, I. A. (2011). Achieving controllability of electric loads. Proceedings of the IEEE, 99, 184-199.

Caramanis, M., Ntakou, E., Hogan, W. W., Chakrabortty, A., \& Schoene, J. (2016). Co-optimization of power and reserves in dynamic T\&D power markets with nondispatchable renewable generation and distributed energy resources. Proceedings of the IEEE, 104, 807-836.

Conejo, A. J., Carrión, M., \& a Morales, J. M. (2010). Decision Making Under Uncertainty in Electricity Markets. Springer.

Conejo, A. J., Castillo, E., Minguez, R., \& Garcia-Bertrand, R. (2006). Decomposition Techniques in Mathematical Programming: Engineering and Science Applications. Springer Science \& Business Media.

Dahleh, M. A., Tahbaz-Salehi, A., Tsitsiklis, J. N., \& Zoumpoulis, S. I. (2016). Coordination with local information. Operations Research, 64, 622-637.

Delikaraoglou, S., \& Pinson, P. (2019). Optimal allocation of HVDC interconnections for exchange of energy and reserve capacity services. Energy Systems, 10, 635-675.

Dempe, S. (2002). Foundations of bilevel programming. Springer Science \& Business Media.

Dvorkin, V., Delikaraoglou, S., \& Morales, J. M. (2019a). Setting reserve requirements to approximate the efficiency of the stochastic dispatch. IEEE Transactions on Power Systems, 34, 1524-1536.

Dvorkin, V., Kazempour, J., \& Pinson, P. (2019b). Electricity market equilibrium under information asymmetry. Operations Research Letters, 47, 521-526.

Dvorkin, Y. (2020). A chance-constrained stochastic electricity market. IEEE Transactions on Power Systems, 35, 2993-3003.

Ehrenmann, A., \& Smeers, Y. (2005). Inefficiencies in European congestion management proposals. Utilities Policy, 13, 135-152.

Farivar, M., \& Low, S. H. (2013). Branch flow model: Relaxations and convexification-Part I. IEEE Transactions on Power Systems, 28, 2554-2564.

Fortuny-Amat, J., \& McCarl, B. (1981). A representation and economic interpretation of a two-level programming problem. The Journal of the Operational Research Society, 32, 783-792.

Frank, S. M., \& Rebennack, S. (2015). Optimal design of mixed AC-DC distribution systems for commercial buildings: A nonconvex generalized Benders decomposition approach. European Journal of Operational Research, 242, 710-729.

Gabriel, S., Conejo, A., Fuller, J., Hobbs, B., \& Ruiz, C. (2012). Complementarity modeling in energy markets. Springer Science \& Business Media. 
Gerard, H., Rivero, E., \& Six, D. (2016). Basic schemes for TSO-DSO coordination and ancillary services provision. Technical Report. URL: http://smartnet-project.eu/wp-content/uploads/2016/12/D1.3_20161202_ V1.0.pdf.

Gerard, H., Rivero Puente, E. I., \& Six, D. (2018). Coordination between transmission and distribution system operators in the electricity sector: A conceptual framework. Utilities Policy, 50, 40-48.

Gonzalez, D. M., Hachenberger, J., Hinker, J., Rewald, F., Rehtanz, C., \& Myrzik, J. (2018). Determination of the time-dependent flexibility of active distribution networks to control their TSO-DSO interconnection power flow. In Power Systems Computation Conference (PSCC 2018) (pp. 1-7). Dublin, Ireland.

Greening, L. A., Greene, D. L., \& Difiglio, C. (2000). Energy efficiency and consumption - the rebound effect-a survey. Energy Policy, 28, $389-401$.

Hadush, S. Y., \& Meeus, L. (2018). DSO-TSO cooperation issues and solutions for distribution grid congestion management. Energy Policy, 120, 610-621.

Heinrich, C., Ziras, C., Jensen, T. V., Bindner, H. W., \& Kazempour, J. (2021). A local flexibility market mechanism with capacity limitation services. Energy Policy, 156, 112335.

Hermann, A., Jensen, T., Kazempour, J., \& Østergaard, J. (2021). Online appendix. URL: https://github.com/ alherm/TSO-DSO_coordination.

Jensen, T. V., Kazempour, J., \& Pinson, P. (2018). Cost-optimal ATCs in zonal electricity markets. IEEE Transactions on Power Systems, 33, 3624-3633.

Kleinert, T., Labbe, M., Plein, F., \& Schmidt, M. (2020). Technical Note-There's no free lunch: On the hardness of choosing a correct Big-M in bilevel optimization. Operations Research, 68, ii-iv.

Laur, A., Nieto Martin, J., Bunn, D. W., \& Vicente Pastor, A. (2020). Optimal procurement of flexibility services within electricity distribution networks. European Journal of Operational Research, 285, 34-47.

Le Cadre, H., Mezghani, I., \& Papavasiliou, A. (2019). A game-theoretic analysis of transmission-distribution system operator coordination. European Journal of Operational Research, 274, 317-339.

Li, X., Tomasgard, A., \& Barton, P. (2011). Nonconvex generalized Benders decomposition for stochastic separable mixed-integer nonlinear programs. Journal of Optimization Theory and Applications, 151, 425-454.

Low, S. H. (2014). Convex relaxation of optimal power flow-Part I: Formulations and equivalence. IEEE Transactions on Control of Network Systems, 1, 15-27.

Luo, Z. Q., Pang, J. S., \& Ralph, D. (1996). Mathematical programs with equilibrium constraints. Cambridge University Press.

Lété, Q., \& Papavasiliou, A. (2021). Impacts of transmission switching in zonal electricity markets - Part I. IEEE Transactions on Power Systems, 36, 902-913.

Maggioni, F., Cagnolari, M., \& Bertazzi, L. (2019). The value of the right distribution in stochastic programming with application to a Newsvendor problem. Computational Management Science, 16, 739-758.

Mitridati, L., Kazempour, J., \& Pinson, P. (2020). Heat and electricity market coordination: A scalable complementarity approach. European Journal of Operational Research, 283, 1107-1123.

Mohammadi, A., Mehrtash, M., \& Kargarian, A. (2019). Diagonal quadratic approximation for decentralized collaborative TSO+DSO optimal power flow. IEEE Transactions on Smart Grid, 10, 2358-2370.

Molzahn, D. K., \& Hiskens, I. A. (2019). A survey of relaxations and approximations of the power flow equations. Foundations and Trends in Electric Energy Systems, 4, 1-221.

Morales, J. M., Zugno, Z., Pineda, S., \& Pinson, P. (2014). Electricity market clearing with improved scheduling of stochastic production. European Journal of Operational Research, 235, 765-774.

Morstyn, T., Teytelboym, A., \& Mcculloch, M. D. (2019). Designing decentralized markets for distribution system flexibility. IEEE Transactions on Power Systems, 34, 2128-2139.

Nick, M., Cherkaoui, R., Le Boudec, J.-Y., \& Paolone, M. (2018). An exact convex formulation of the optimal power flow in radial distribution networks including transverse components. IEEE Transactions on Automatic Control, 63, 682-697.

Ordoudis, C., Delikaraoglou, S., Kazempour, J., \& Pinson, P. (2020). Market-based coordination for integrated electricity and natural gas systems under uncertain supply. European Journal of Operational Research, 287, 1105-1119.

Ordoudis, C., Pinson, P., Morales, J. M., \& Zugno, M. (2016). An updated version of the IEEE RTS 24-bus system for electricity market and power system operation studies. Technical University of Denmark Report, (pp. 1-6). URL: http://orbit.dtu.dk/files/120568114/An.

Pineda, P., \& Morales, J. M. (2019). Solving linear bilevel problems using big-Ms: Not all that glitters is gold. IEEE Transactions on Power Systems, 34, 2469-2471.

Pinson, P., Madsen, H., Nielsen, H. A., Papaefthymiou, G., \& Klockl, B. (2009). From probabilistic forecasts to 
statistical scenarios of short-term wind power production. Wind Energy, 12, 51-62.

Pozo, D., Sauma, E., \& Contreras, J. (2017). Basic theoretical foundations and insights on bilevel models and their applications to power systems. Annals of Operations Research, 254, 303-334.

Rahmaniani, R., Crainic, T. G., Gendreau, M., \& Rei, W. (2017). The Benders decomposition algorithm: A literature review. European Journal of Operational Research, 259, 801-817.

Ruszczynski, A., \& Swietanowski, A. (1997). Accelerating the regularized decomposition method for two stage stochastic linear problems. European Journal of Operational Research, 101, 328-342.

Schwele, A., Ordoudis, C., Pinson, P., \& Kazempour, J. (2021). Coordination of power and natural gas markets via financial instruments. Computational Management Science, . To be published, DOI: 10.1007/s10287-021-00403-x.

Silva, J. P., Sumaili, J. A., Bessa, R. J., Seca, L., Matos, M. A., Miranda, V., Caujolle, M., Goncer-Maraver, B., \& Sebastian-Viana, M. (2018). Estimating the active and reactive power flexibility area at the TSO-DSO interface. IEEE Transactions on Power Systems, 33, 4741-4750.

Skar, C., Doorman, G., \& Tomasgard, A. (2014). Large-scale power system planning using enhanced Benders decomposition. Power Systems Computation Conference (PSCC 2014), (pp. 1-7).

Ströhle, P., \& Flath, C. M. (2016). Local matching of flexible load in smart grids. European Journal of Operational Research, 253, 811-824.

Taylor, J. A. (2015). Convex optimization of power systems. Cambridge University Press.

The SMARTNET Project Final Results (2019). TSO-DSO Coordination for Acquiring Ancillary Services from Distribution Grids. Technical Report. URL: http://smartnet-project.eu/wp-content/uploads/2019/05/ SmartNet-Booktlet.pdf.

Trukhanov, S., Ntaimo, L., \& Schaefer, A. (2010). Adaptive multicut aggregation for two-stage stochastic linear programs with recourse. European Journal of Operational Research, 206, 395-406.

Verzijlbergh, R. A., De Vries, L. J., \& Lukszo, Z. (2014). Renewable energy sources and responsive demand. Do we need congestion management in the distribution grid? IEEE Transactions on Power Systems, 29, 2119-2128.

Vicente Pastor, A., Nieto Martin, J., Bunn, D. W., \& Laur, A. (2019). Evaluation of flexibility markets for retailerDSO-TSO coordination. IEEE Transactions on Power Systems, 34, 2003-2012.

Villar, J., Bessa, R., \& Matos, M. (2018). Flexibility products and markets: Literature review. Electric Power Systems Research, 154, 329-340.

You, F., \& Grossmann, I. (2013). Multicut Benders decomposition algorithm for process supply chain planning under uncertainty. Annals of Operations Research, 210, 191-211.

Yuan, Z., \& Hesamzadeh, M. R. (2017). Hierarchical coordination of TSO-DSO economic dispatch considering large-scale integration of distributed energy resources. Applied Energy, 195, 600-615.

Zhang, C., Ding, Y., Nordentoft, N. C., Pinson, P., \& Østergaard, J. (2014). FLECH: A Danish market solution for DSO congestion management through DER flexibility services. Journal of Modern Power Systems and Clean Energy, 2, 126-133.

Zhao, J., Zheng, T., \& Litvinov, E. (2016). A unified framework for defining and measuring flexibility in power system. IEEE Transactions on Power Systems, 31, 339-347.

Zohrizadeh, F., Josz, C., Jin, M., Madani, R., Lavaei, J., \& Sojoudi, S. (2020). A survey on conic relaxations of optimal power flow problem. European Journal of Operational Research, 287, 391-409.

Østergaard, J., Ziras, C., Bindner, H. W., Kazempour, J., Marinelli, M., Markussen, P., Rosted, S. H., \& Christensen, J. S. (2021). Energy security through demand-side flexibility: The case of Denmark. IEEE Power and Energy Magazine, 19, 46-55. 